\title{
Fundamental solution of the time-fractional telegraph Dirac operator*
}

\author{
M. Ferreira ${ }^{\dagger, \ddagger} \quad$ M.M. Rodrigues ${ }^{\ddagger} \quad$ N. Vieira ${ }^{\ddagger}$ \\ † School of Technology and Management, \\ Polytechnic Institute of Leiria \\ P-2411-901, Leiria, Portugal. \\ ‡ CIDMA - Center for Research and Development in Mathematics and Applications \\ Department of Mathematics, University of Aveiro \\ Campus Universitário de Santiago, 3810-193 Aveiro, Portugal. \\ E-mails: milton.ferreira@ipleiria.pt, mrodrigues@ua.pt, nloureirovieira@gmail.com
}

\begin{abstract}
In this work we obtain the fundamental solution (FS) of the multidimensional time-fractional telegraph Dirac operator where the two time-fractional derivatives of orders $\alpha \in] 0,1]$ and $\beta \in] 1,2]$ are in the Caputo sense. Explicit integral and series representation of the FS are obtained for any dimension. We present and discuss some plots of the FS for some particular values of the dimension and of the fractional parameters $\alpha$ and $\beta$. Finally, using the FS we study some Poisson and Cauchy problems.
\end{abstract}

Keywords: Time-fractional telegraph and telegraph Dirac operators; Fundamental solution; Caputo fractional derivative; Multivariate Mittag-Leffler functions; H-function of two variables.

MSC 2010: 30G35, 35R11, 26A33, 35A08, 35C15.

\section{Introduction}

In contrast to the wave equation, the telegraph equation has the potential to describe both diffusive and wavelike phenomena, due to the simultaneous presence of first and second order time derivatives. Telegraph equations have an extraordinary importance in electrodynamics (the scalar Maxwell equations are of this type), in the theory of damped vibrations and in probability because they are connected with finite velocity random motions.

In the last years, the interest in the study of fractional differential equations has increased considerably due essentially to the wide range of applications. These type of equations are used mostly to model processes in the fields of engineering, viscoelastic materials, hydrology, system control, just to mention some (see [16,21]). The use of fractional derivatives in the telegraph equation give us the possibility of describing memory and heredity properties of telegraph processes (see $[16,21]$ ).

The aim of this paper is to obtain explicit integral and series representations for the FS of the so-called time-fractional telegraph Dirac operator. The time-fractional telegraph Dirac operator is a combination of the Dirac operator in space with a sum of two fractional derivatives of orders $\alpha \in] 0,1]$ and $\beta \in] 1,2]$ in time, written using a Witt basis in the framework of Clifford algebra. This operator factorizes the time-fractional telegraph operator, which is a sum of the Laplace operator with the two time-fractional derivatives already mentioned. The FS of this operator was studied recently in [11]. The FS of the time-fractional telegraph Dirac operator can be seen as a refinement of the FS of the time-fractional telegraph operator. This opens new possibilities for the

${ }^{*}$ The final version is published in Mathematical Methods in the Applied Sciences, 40-No.18, (2017), 7033-7050. It as available via the website http://onlinelibrary.wiley.com/doi/10.1002/mma.4511/full 
development of a fractional function theory for this operator in the context of Clifford analysis. The geometric nature of Clifford algebras allows the resolution of PDEs using the geometric properties of the domain where the differential operator acts (see [4-6]). Connections between Clifford analysis and fractional calculus were recently established in the study of the stationary fractional Dirac operator (see $[9,10,15,22])$ and in the study of the time-fractional diffusion wave operator (see [8]).

The structure of the paper reads as follows: in the preliminaries section we recall some basic concepts about fractional calculus, special functions, Clifford analysis and the Witt basis. In Section 3 we recall the integral and series representations for the FS of the time-fractional telegraph operator in $\mathbb{R}^{n} \times \mathbb{R}^{+}$obtained in [11]. In Section 4, we derive the FS for the time-fractional telegraph Dirac operator in the form of integral and series from the representations presented in Section 3. We remark that the series representation depends on the parity of the space dimension, as it happens in [11]. Connections with the time-fractional parabolic Dirac operator are established in Section 4. In Section 5 we present and discuss some plots of the FS obtained in Section 4 for some particular values of the dimension and of the order of the fractional derivatives. In Section 6 we study some Poisson and Cauchy problems related with the time-fractional telegraph operators described in the paper.

\section{Preliminaries}

\subsection{Special functions and basic results}

In this section we present the main tools concerning fractional derivatives and special functions that we will use in our analysis. We start by recalling some properties of the Gamma function that are used in this paper:

$$
\begin{aligned}
& \Gamma(z+n)=(z)_{n} \Gamma(z), \quad n \in \mathbb{N} \\
& \Gamma(z) \Gamma(-z)=\frac{-\pi}{z \sin (\pi z)} \\
& \Gamma\left(\frac{1}{2}-z\right)=\frac{\pi}{\cos (\pi z) \Gamma\left(\frac{1}{2}+z\right)} .
\end{aligned}
$$

In $(1),(z)_{n}$ denotes the Pochhammer symbol. An important function that appears in this work is the multivariate Mittag-Leffler function.

Definition 2.1 (see [18]) The multivariate Mittag-Leffler function $E_{\left(a_{1}, \ldots, a_{n}\right), b}\left(z_{1}, \ldots, z_{n}\right)$ of $n$ complex variables $z_{1}, \ldots, z_{n} \in \mathbb{C}$ with complex parameters $a_{1}, \ldots, a_{n} \in \mathbb{C}$ (with positive real parts) is defined by

$$
E_{\left(a_{1}, \ldots, a_{n}\right), b}\left(z_{1}, \ldots, z_{n}\right)=\sum_{k=0}^{+\infty} \sum_{\substack{l_{1}+\ldots+l_{n}=k \\
l_{1}, \ldots, l_{n} \geq 0}}\left(\begin{array}{c}
k \\
l_{1}, \ldots, l_{n}
\end{array}\right) \frac{\prod_{i=1}^{n} z_{i}^{l_{i}}}{\Gamma\left(b+\sum_{i=1}^{n} a_{i} l_{i}\right)},
$$

where the multinomial coefficients are given by

$$
\left(\begin{array}{c}
k \\
l_{1}, \ldots, l_{n}
\end{array}\right):=\frac{k !}{l_{1} ! \times \ldots \times l_{n} !} .
$$

When $n=2$ the multivariate Mittag-Leffler function (4) can be written as

$$
\begin{aligned}
E_{\left(a_{1}, a_{2}\right), b}\left(z_{1}, z_{2}\right) & =\sum_{k=0}^{+\infty} \sum_{\substack{l_{1}+l_{2}=k \\
l_{1}, l_{2} \geq 0}} \frac{k !}{l_{1} ! l_{2} !} \frac{z_{1}^{l_{1}} z_{2}^{l_{2}}}{\Gamma\left(b+a_{1} l_{1}+a_{2} l_{2}\right)} \\
& =\sum_{l_{1}=0}^{+\infty} \sum_{l_{2}=0}^{+\infty} \frac{\left(l_{1}+l_{2}\right) !}{l_{1} ! l_{2} !} \frac{z_{1}^{l_{1}} z_{2}^{l_{2}}}{\Gamma\left(b+a_{1} l_{1}+a_{2} l_{2}\right)} .
\end{aligned}
$$

When $n=1$ the multivariate Mittag-Leffler function (4) reduces to the two-parameter Mittag-Leffler function

$$
E_{a_{1}, b}\left(z_{1}\right)=\sum_{k=0}^{\infty} \frac{z_{1}^{k}}{\Gamma\left(b+k a_{1}\right)} .
$$

For general properties of the Mittag-Leffler function see $[14,18]$. 
Definition 2.2 (see [12]) The Fox H-function $H_{p, q}^{m, n}$ is defined via a Mellin-Barnes type integral of the form

$$
H_{p, q}^{m, n}\left[z \mid \begin{array}{c}
\left(a_{1}, \alpha_{1}\right), \ldots,\left(a_{p}, \alpha_{p}\right) \\
\left(b_{1}, \beta_{1}\right), \ldots,\left(b_{q}, \beta_{q}\right)
\end{array}\right]=\frac{1}{2 \pi i} \int_{\mathcal{L}} \frac{\prod_{j=1}^{m} \Gamma\left(b_{j}+\beta_{j} s\right) \prod_{i=1}^{n} \Gamma\left(1-a_{i}-\alpha_{i} s\right)}{\prod_{i=n+1}^{p} \Gamma\left(a_{i}+\alpha_{i} s\right) \prod_{j=m+1}^{q} \Gamma\left(1-b_{j}-\beta_{j} s\right)} z^{-s} d s
$$

where $m, n, p, q \in \mathbb{N}$ are such that $0 \leq m \leq q, 0 \leq n \leq p, a_{i}, b_{j} \in \mathbb{C}$, and $\alpha_{i}, \beta_{j} \in \mathbb{R}^{+}(i=1,2, \ldots, p ; j=$ $1,2, \ldots, q)$.

The conditions for the existence of the Fox H-function and the orientation of the contour $\mathcal{L}$ are given by Theorem 1.1 in [17]. The H-function can be generalized for $r$ complex variables (see [2]). For the purpose of this work we present only the definition of the H-function of two complex variables.

Definition 2.3 (see [2]) The H-function of two complex variables is defined via a Mellin-Barnes type integral of the form

$$
\begin{array}{r}
H[x, y]=H_{p_{1}, q_{1} ; p_{2}, q_{2} ; p_{3}, q_{3}}^{0, n_{1} ; m_{2}, n_{2} ; m_{3}, n_{3}}\left[\begin{array}{c|c}
x & \left(a_{j} ; \alpha_{j}, A_{j}\right)_{1, p_{1}} ;\left(c_{j}, \gamma_{j}\right)_{1, p_{2}} ;\left(e_{j}, E_{j}\right)_{1, p_{3}} \\
y & \left(b_{j} ; \beta_{j}, B_{j}\right)_{1, q_{1}} ;\left(d_{j}, \delta_{j}\right)_{1, q_{2}} ;\left(f_{j}, F_{j}\right)_{1, q_{3}}
\end{array}\right] \\
=\frac{1}{(2 \pi i)^{2}} \int_{\mathcal{L}_{2}} \int_{\mathcal{L}_{1}} \phi(s, w) \phi_{1}(s) \phi_{2}(w) x^{s} y^{w} d s d w
\end{array}
$$

where

$$
\begin{aligned}
& \phi(s, w)=\frac{\prod_{i=1}^{n_{1}} \Gamma\left(1-a_{i}+\alpha_{i} s+A_{i} w\right)}{\prod_{i=n_{1}+1}^{p_{1}} \Gamma\left(a_{i}-\alpha_{i} s-A_{i} w\right) \prod_{j=1}^{q_{1}} \Gamma\left(1-b_{j}+\beta_{j} s+B_{j} w\right)}, \\
& \phi_{1}(s)=\frac{\prod_{j=1}^{m_{2}} \Gamma\left(d_{j}-\delta_{j} s\right) \prod_{i=1}^{n_{2}} \Gamma\left(1-c_{i}+\gamma_{i} s\right)}{\prod_{j=m_{2}+1}^{q_{2}} \Gamma\left(1-d_{j}+\delta_{j} s\right) \prod_{i=n_{2}+1}^{p_{2}} \Gamma\left(c_{i}-\gamma_{i} s\right)}, \\
& \phi_{2}(w)=\frac{\prod_{j=1}^{m_{3}} \Gamma\left(f_{j}-F_{j} w\right) \prod_{i=1}^{n_{3}} \Gamma\left(1-e_{i}+E_{i} w\right)}{\prod_{j=m_{3}+1}^{q_{3}} \Gamma\left(1-f_{j}+F_{j} w\right) \prod_{i=n_{3}+1}^{p_{3}} \Gamma\left(e_{i}-E_{i} w\right)},
\end{aligned}
$$

and where an empty product is interpreted as $1, x, y \in \mathbb{C}, m_{i}, n_{i}, p_{i}, q_{i} \in \mathbb{Z}$ such that $0 \leq m_{i} \leq q_{i}, 0 \leq n_{i} \leq p_{i}$ $(i=1,2,3) ; a_{i}, b_{j}, c_{i}, d_{j}, e_{i}, f_{j} \in \mathbb{C}, \alpha_{i}, A_{i}, \beta_{j}, B_{j}, \gamma_{i}, \delta_{j}, E_{i}, F_{j} \in \mathbb{R}^{+}$and the sequence of parameters $\left(a_{j}\right),\left(b_{j}\right)$, $\left(c_{j}\right),\left(d_{j}\right),\left(e_{j}\right)$ and $f_{j}$ are restricted that none of the poles of the integrand coincide. The contour $\mathcal{L}_{1}$ in the complex s-plane, and the contour $\mathcal{L}_{2}$ in the complex w-plane, are of the Mellin-Barnes type with indentations, if necessary, to ensure that they separate one set of poles from the other.

In $[2]$ the author proved that if

$$
\begin{aligned}
& \Omega_{1}=\sum_{i=1}^{n_{1}} \alpha_{i}-\sum_{i=n_{1}+1}^{p_{1}} \alpha_{i}-\sum_{i=1}^{q_{1}} \beta_{i}+\sum_{i=1}^{m_{2}} \delta_{i}-\sum_{j=m_{2}+1}^{q_{2}} \delta_{i}+\sum_{i=1}^{n_{2}} \gamma_{i}-\sum_{i=n_{2}+1}^{p_{2}} \gamma_{i} \geq 0 \\
& \Omega_{2}=\sum_{i=1}^{n_{1}} A_{i}-\sum_{i=n_{1}+1}^{p_{1}} A_{i}-\sum_{i=1}^{q_{1}} B_{i}+\sum_{i=1}^{m_{3}} F_{i}-\sum_{j=m_{3}+1}^{q_{3}} F_{i}+\sum_{i=1}^{n_{3}} E_{i}-\sum_{i=n_{3}+1}^{p_{3}} E_{i} \geq 0,
\end{aligned}
$$

and with the points $x=0$ and $y=0$ being tacitly excluded, the double Mellin-Barnes integral converges absolutely inside the sector given by

$$
|\arg (x)|<\frac{\pi}{2} \Omega_{1}, \quad|\arg (y)|<\frac{\pi}{2} \Omega_{2}
$$

When all poles of the integrand are simple, we have the following asymptotic behaviour for large values of $x$ and $y(\operatorname{see}[1])$

$$
H[x, y]=O\left(|x|^{\beta_{1}},|y|^{\beta_{2}}\right)
$$

where

$$
\begin{array}{ll}
\beta_{1}=\max \left\{\Re\left(\frac{c_{j}-1}{\delta_{j}}\right)\right\}, & j=1,2, \ldots, n_{2}, \\
\beta_{2}=\max \left\{\Re\left(\frac{e_{j}-1}{E_{j}}\right)\right\}, & j=1,2, \ldots, n_{3} .
\end{array}
$$

Moreover, for $E_{\left(a_{1}, a_{2}\right), b}$ we have the following two results (see [11]) 
Lemma 2.4 The multivariate Mittag-Leffler function $E_{\left(a_{1}, a_{2}\right), b}$ has the following representation in the form of double Mellin-Barnes integral

$$
E_{\left(a_{1}, a_{2}\right), b}\left(z_{1}, z_{2}\right)=\frac{1}{(2 \pi i)^{2}} \int_{\mathcal{L}_{1}} \int_{\mathcal{L}_{2}} \frac{\Gamma(1+s+w) \Gamma(-s) \Gamma(-w)}{\Gamma\left(b+a_{1} w+a_{2} s\right)} z_{2}^{s} z_{1}^{w} d w d s .
$$

Corollary 2.5 The multivariate Mittag-Leffler function $E_{\left(a_{1}, a_{2}\right), b}$ has the following representation in the form of Fox H-function of two variables

$$
E_{\left(a_{1}, a_{2}\right), b}\left(z_{1}, z_{2}\right)=H_{1,1 ; 0,1 ; 0,1}^{0,1 ; 1,0 ; 1,0}\left[\begin{array}{c|c}
z_{2} & (0 ; 1,1) ;-;- \\
z_{1} & \left(1-b ; a_{1}, a_{2}\right) ;(0,1) ;(0,1)
\end{array}\right] .
$$

In (9) and throughout the paper a horizontal line in the H-function means the absence of parameters. Now we recall the definition of the spaces $C_{\alpha}, \alpha \in \mathbb{R}$, and $C_{\alpha}^{m}, m \in \mathbb{N}$, given in [18].

Definition 2.6 (see [18]) A real or complex-valued function $f(t), t>0$, is said to be in the space $C_{\alpha}, \alpha \in \mathbb{R}$, if there exists a real number $p>\alpha$ such that $f(t)=t^{p} f_{1}(t)$ for some function $f_{1} \in C[0, \infty)$.

It is easy to see that $C_{\alpha}$ is a vector space and the set of spaces $C_{\alpha}$ is ordered by inclusion according to $C_{\alpha} \subseteq C_{\beta} \Leftrightarrow \alpha \geq \beta$.

Definition 2.7 (see [18]) A function $f(t), t>0$, is said to be in the space $C_{\alpha}^{m}, m \in \mathbb{N}$, if and only if $f^{(m)} \in C_{\alpha}$.

The next theorem will be used in our analysis and allow us to solve general linear differential equations with constant coefficients and Caputo derivatives.

Theorem 2.8 (see [18, Thm 4.1]) Let $\mu>\mu_{1}>\ldots>\mu_{n} \geq 0, m_{i}-1<\mu_{i} \leq m_{i}, m_{i} \in \mathbb{N}, \lambda_{i} \in \mathbb{R}, i=1, \ldots, n$. Consider the initial value problem

$$
\left\{\begin{array}{l}
D_{t}^{\mu} y(t)-\sum_{i=1}^{n} \lambda_{i} D_{t}^{\mu_{i}} y(t)=g(t) \\
y^{(k)}(0)=c_{k} \in \mathbb{R}, \quad k=0, \ldots, m-1, m-1<\mu \leq m,
\end{array}\right.
$$

where $g$ is assumed to lie in $C_{-1}$ if $\mu \in \mathbb{N}_{0}$ or in $C_{-1}^{1}$ if $\mu \notin \mathbb{N}_{0}$. Then (10) has a unique solution in the space $C_{-1}^{m}$ of the form

$$
y(t)=\int_{0}^{t} s^{\mu-1} E_{\left(\mu-\mu_{1}, \ldots, \mu-\mu_{n}\right), \mu}\left(\lambda_{1} s^{\mu-\mu_{1}}, \ldots, \lambda_{1} s^{\mu-\mu_{n}}\right) g(t-s) d s+\sum_{k=0}^{m-1} c_{k} u_{k}(t), \quad t \geq 0,
$$

with

$$
u_{k}(t)=\frac{t^{k}}{k !}+\sum_{i=l_{k}+1}^{n} \lambda_{i} t^{k+\mu-\mu_{i}} E_{\left(\mu-\mu_{1}, \ldots, \mu-\mu_{n}\right), k+1+\mu-\mu_{i}}\left(\lambda_{1} t^{\mu-\mu_{1}}, \ldots, \lambda_{n} t^{\mu-\mu_{n}}\right), \quad k=0, \ldots, m-1 .
$$

The natural numbers $l_{k}, k=0, \ldots, m-1$ are determined from the conditions $m_{l_{k}} \geq k+1$ and $m_{l_{k}+1} \leq k$. In the case $m_{i} \leq k, i=0, \ldots, m-1$, we set $l_{k}:=0$, and of $m_{i} \geq k+1, i=0, \ldots, m-1$, then $l_{k}:=n$.

\subsection{Clifford analysis}

We consider the $n$-dimensional vector space $\mathbb{R}^{n}$ endowed with an orthonormal basis $\left\{e_{1}, \cdots, e_{n}\right\}$. We define the universal real Clifford algebra $C \ell_{0, n}$ as the $2^{n}$-dimensional associative algebra which obeys the multiplication rule

$$
e_{i} e_{j}+e_{j} e_{i}=-2 \delta_{i, j}, \quad i, j=1, \ldots, n
$$

A vector space basis for $C \ell_{0, n}$ is generated by the elements $e_{0}=1$ and $e_{A}=e_{h_{1}} \cdots e_{h_{k}}$, where $A=$ $\left\{h_{1}, \ldots, h_{k}\right\} \subset M=\{1, \ldots, n\}$, for $1 \leq h_{1}<\cdots<h_{k} \leq n$. Each element $x \in C \ell_{0, n}$ can be represented by 
$x=\sum_{A} x_{A} e_{A}$, with $x_{A} \in \mathbb{R}$. The Clifford conjugation is defined by $\overline{1}=1, \overline{e_{j}}=-e_{j}$ for all $j=1, \ldots, n$, and we have $\overline{a b}=\bar{b} \bar{a}$. We introduce the complexified Clifford algebra $\mathbb{C}_{n}$ as the tensor product

$$
\mathbb{C}_{n}:=\mathbb{C} \otimes C \ell_{0, n}=\left\{w=\sum_{A} w_{A} e_{A}, w_{A} \in \mathbb{C}, A \subset M\right\},
$$

where the imaginary unit $i$ of $\mathbb{C}$ commutes with the basis elements, i.e., $i e_{j}=e_{j} i$ for all $j=1, \ldots, n$. To avoid ambiguities with the Clifford conjugation, we denote the complex conjugation by $\sharp$, in the sense that for a complex scalar $w_{A}=a_{A}+i b_{A}$ we have that $w_{A}^{\sharp}=a_{A}-i b_{A}$. The complex conjugation can be extended linearly to whole of the Clifford algebras and leaves the elements $e_{j}$ invariant, i.e., $e_{j}^{\sharp}=e_{j}$ for all $j=1, \ldots, n$. We also have a pseudonorm on $\mathbb{C}_{n}$ defined by $|w|:=\sum_{A}\left|w_{A}\right|$ where $w=\sum_{A} w_{A} e_{A}$. Notice also that for $a, b \in \mathbb{C}_{n}$ we only have $|a b| \leq 2^{n}|a||b|$.

A $\mathbb{C}_{n}$-valued function defined on an open set $U \subseteq \mathbb{R}^{n}$ has the representation $f=\sum_{A} f_{A} e_{A}$ with $\mathbb{C}$-valued components $f_{A}$. Properties such as continuity and differentiability needs to be understood component-wisely. Next, we introduce the Euclidean Dirac operator $D_{x}=\sum_{i=1}^{n} e_{i} \partial_{x_{i}}$, which factorizes the $n$-dimensional Euclidean Laplacian, i.e., $D_{x}^{2}=-\Delta=-\sum_{i=1}^{n} \partial_{x_{i}}^{2}$. A Clifford valued $C^{1}$-function $f$ is called left-monogenic if it satisfies $D_{x} f=0$ on $U$ (resp. right-monogenic if it satisfies $f D_{x}=0$ on $U$ ).

In order to introduce a Witt basis to define a parabolic Dirac operator we embed $\mathbb{R}^{n}$ into $\mathbb{R}^{n+2}$ and consider two new elements $e_{+}$and $e_{-}$such that $e_{+}^{2}=+1, e_{-}^{2}=-1$, and $e_{+} e_{-}=-e_{-} e_{+}$. Moreover, $e_{+}$and $e_{-}$ anticommute with all the basis elements $e_{i}, i=1, \ldots, n$. Hence, $\left\{e_{1}, \ldots, e_{n}, e_{+}, e_{-}\right\}$spans $\mathbb{R}^{n+1,1}$. With the elements $e_{+}$and $e_{-}$we construct two nilpotent elements $\mathfrak{f}$ and $\mathfrak{f}^{\dagger}$ given by

$$
\mathfrak{f}=\frac{e_{+}-e_{-}}{2} \text { and } \mathfrak{f}^{\dagger}=\frac{e_{+}+e_{-}}{2} .
$$

These elements satisfy the following relations

$$
(\mathfrak{f})^{2}=\left(\mathfrak{f}^{+}\right)^{2}=0, \quad \mathfrak{f f}^{+}+\mathfrak{f}^{+} \mathfrak{f}=1, \quad \mathfrak{f} e_{j}+e_{j} \mathfrak{f}=\mathfrak{f}^{+} e_{j}+e_{j} \mathfrak{f}^{+}=0, j=1, \ldots, n .
$$

The extended basis $\left\{e_{1}, \ldots, e_{n}, \mathfrak{f}, \mathfrak{f}^{\dagger}\right\}$ allow us to define the telegraph Dirac operator as $D_{x, t}:=D_{x}+\mathfrak{f}\left(A \partial_{t}^{2}+B \partial_{t}\right)+$ $\mathfrak{f}^{\dagger}$, where $D_{x}$ stands for the Dirac operator in $\mathbb{R}^{n}$, and $A, B \in \mathbb{R}$ with $A \neq 0$. The operator $D_{x, t}$ acts on $\mathbb{C}_{n}$-valued functions defined on time dependent domains $\Omega \times I \subseteq \mathbb{R}^{n} \times \mathbb{R}^{+}$, i.e., functions in the variables $\left(x_{1}, x_{2}, \ldots, x_{n}, t\right)$ where $x_{i} \in \mathbb{R}$ for $i=1, \ldots, n$, and $t \in \mathbb{R}^{+}$. For the sake of readability, we abbreviate the space-time tuple $\left(x_{1}, x_{2}, \ldots, x_{n}, t\right)$ simply by $(x, t)$, assigning $x=x_{1} e_{1}+\cdots+x_{n} e_{n}$. For additional details about Clifford analysis and the Witt basis, we refer the interested reader for instance to $[6,7,13]$.

\section{Fundamental solution of the time-fractional telegraph operator}

In this section we recall some results concerning the FS of the time-fractional telegraph operator, which was deduced in [11]. We look for the function $G_{n}^{\alpha, \beta}(x, t)$ that satisfies the following Cauchy problem:

$$
\left\{\begin{array}{l}
\left(D_{t}^{\beta}+a D_{t}^{\alpha}-c^{2} \Delta_{x}\right) G_{n}^{\alpha, \beta}(x, t)=0 \\
G_{n}^{\alpha, \beta}(x, 0)=\delta(x) \\
\frac{\partial G_{n}^{\alpha, \beta}}{\partial t}(x, 0)=0
\end{array}\right.
$$

where $x \in \mathbb{R}^{n}, t>0,1<\beta \leq 2,0<\alpha \leq 1, a \geq 0, c>0, \delta$ is the delta Dirac function in $\mathbb{R}^{n}$, and $\Delta_{x}$ is the Laplace operator in $\mathbb{R}^{n}$. For $\gamma>0, D_{t}^{\gamma}$ is the Caputo fractional derivative of order $\gamma$ defined by:

$$
D_{t}^{\gamma} u(t)=\left\{\begin{array}{cl}
\frac{1}{\Gamma(m-\gamma)} \int_{0}^{t}(t-s)^{-\gamma+m-1} u^{(m)}(s) d s, & m-1<\gamma<m \\
u^{(m)}(t), & \gamma=m
\end{array}\right.
$$


where $u^{(m)}:=\frac{d^{m} u}{d t^{m}}, m \in \mathbb{N}$ (see [3]). Applying the Fourier transform in $\mathbb{R}^{n}$ to (13) we get the following initial-value problem:

$$
\left\{\begin{array}{l}
\left(D_{t}^{\beta}+a D_{t}^{\alpha}+c^{2}|\kappa|^{2}\right) \widehat{G}_{n}^{\alpha, \beta}(\kappa, t)=0 \\
\widehat{G}_{n}^{\alpha, \beta}(\kappa, 0)=1 \\
\frac{\partial \widehat{G}_{n}^{\alpha, \beta}}{\partial t}(\kappa, 0)=0
\end{array} .\right.
$$

To solve the problem (15) we apply Theorem 2.8 with $\lambda_{1}=-a, \lambda_{2}=-c^{2}|\kappa|^{2}, \mu=\beta, \mu_{1}=\alpha, \mu_{2}=0, n=2$, $m=2, g(t)=0$, obtaining the following solution:

$$
\widehat{G}_{n}^{\alpha, \beta}(\kappa, t)=1-c^{2}|\kappa|^{2} t^{\beta} E_{(\beta-\alpha, \beta), 1+\beta}\left(-a t^{\beta-\alpha},-c^{2}|\kappa|^{2} t^{\beta}\right) .
$$

The multivariate Mittag-Leffler function $E_{(\beta-\alpha, \beta), 1+\beta}$ can be represented in the form of a double series (see $(5))$ :

$$
E_{(\beta-\alpha, \beta), 1+\beta}\left(-a t^{\beta-\alpha},-c^{2}|\kappa|^{2} t^{\beta}\right)=\sum_{j=0}^{+\infty} \sum_{k=0}^{+\infty} \frac{\Gamma(1+k+j)}{\Gamma(1+\beta+(\beta-\alpha) k+\beta j) k ! j !}\left(-a t^{\beta-\alpha}\right)^{k}\left(-c^{2}|\kappa|^{2} t^{\beta}\right)^{j} .
$$

Moreover, it can be written in terms of a double Mellin-Barnes integral and a Fox H-function of two variables (see Lemma 2.4 and Corollary 2.5):

$$
\begin{aligned}
& E_{(\beta-\alpha, \beta), 1+\beta}\left(-a t^{\beta-\alpha},-c^{2}|\kappa|^{2} t^{\beta}\right) \\
& \quad=\frac{1}{(2 \pi i)^{2}} \int_{\mathcal{L}_{1}} \int_{\mathcal{L}_{2}} \frac{\Gamma(1+s+w) \Gamma(-s) \Gamma(-w)}{\Gamma(1+\beta+(\beta-\alpha) w+\beta s)}\left(c^{2}|\kappa|^{2} t^{\beta}\right)^{s}\left(a t^{\beta-\alpha}\right)^{w} d w d s \\
& \quad=H_{1,1 ; 0,1 ; 0,1}^{0,1 ; 1,0 ; 1,0}\left[\begin{array}{c|c}
a t^{\beta-\alpha} & (0 ; 1,1) ;-;- \\
c^{2}|\kappa|^{2} t^{\beta} & (-\beta ; \beta-\alpha, \beta) ;(0,1) ;(0,1)
\end{array}\right] .
\end{aligned}
$$

From the application of the inverse Fourier transform to (16) and taking into account (17) we get (see [11] for more details)

$$
\begin{aligned}
& G_{n}^{\alpha, \beta}(x, t)=\frac{|x|^{1-\frac{n}{2}}}{(2 \pi)^{\frac{n}{2}}} \int_{0}^{+\infty} \tau^{\frac{n}{2}} \widehat{G}_{n}^{\alpha, \beta}(\tau, t) J_{\frac{n}{2}-1}(\tau|x|) d \tau \\
& \quad=\delta(x)-\frac{4 c^{2} t^{\beta}}{|x|^{n+2} \pi^{\frac{n}{2}}} \frac{1}{(2 \pi i)^{2}} \int_{\mathcal{L}_{1}} \int_{\mathcal{L}_{2}} \frac{\Gamma(-s) \Gamma(-w) \Gamma(1+s+w) \Gamma\left(1+\frac{n}{2}+s\right)}{\Gamma(1+\beta+(\beta-\alpha) w+\beta s) \Gamma(-1-s)}\left(\frac{4 c^{2} t^{\beta}}{|x|^{2}}\right)^{s}\left(a t^{\beta-\alpha}\right)^{w} d w d s .
\end{aligned}
$$

In [11] the authors studied the convergence of (19), and obtained its series representation using the residue theory taking into account the parity of the dimension, which is stated in the following theorem.

Theorem 3.1 For $n$ odd, $1<\beta \leq 2$ and $0<\alpha \leq 1$ the FS of the time-fractional telegraph operator is given by

$$
\begin{aligned}
G_{n}^{\alpha, \beta}(x, t)= & \delta(x)-\frac{1}{\pi^{\frac{n}{2}}|x|^{n}} \sum_{k=0}^{+\infty} \frac{\left(-a t^{\beta-\alpha}\right)^{k}}{k !}\left[\sum_{q=0}^{\frac{n-2 k-1}{2}} \frac{(-1)^{q}}{q !} \frac{\Gamma\left(\frac{n}{2}-q-k\right)(q+k)}{\Gamma(1-\beta q-\alpha k)}\left(\frac{|x|^{2}}{4 c^{2} t^{\beta}}\right)^{q+k}\right. \\
& \left.+\frac{(-1)^{\frac{n-1}{2}} \sqrt{\pi}|x|^{n}}{\left(4 c^{2} t^{\beta}\right)^{\frac{n}{2}}} \sum_{p=0}^{+\infty} \frac{\left(\frac{p+n}{2}\right)\left(1-\frac{p+n}{2}\right)_{k-1}}{\left(\frac{p+1}{2}\right)_{\frac{n-1}{2}} \Gamma\left(1+(\beta-\alpha) k-\frac{\beta(p+n)}{2}\right) p !}\left(-\frac{|x|}{c t^{\frac{\beta}{2}}}\right)^{p}\right] .
\end{aligned}
$$


For $n$ even, $1<\beta \leq 2$ and $0<\alpha \leq 1$ the FS of the time-fractional telegraph operator is given by

$$
\begin{aligned}
G_{n}^{\alpha, \beta}(x, t)= & \delta(x)-\frac{4 c^{2} t^{\beta}}{\pi^{\frac{n}{2}}|x|^{n+2}}\left\{\sum _ { k = 0 } ^ { \frac { n } { 2 } - 1 } \frac { ( - a t ^ { \beta - \alpha } ) ^ { k } } { k ! } \left[\sum_{i=0}^{\frac{n}{2}-k-1} \frac{(-1)^{i}}{i !} \frac{\Gamma\left(-i-k+\frac{n}{2}\right)(i+k)}{\Gamma(1-\beta i-\alpha k)}\left(\frac{|x|^{2}}{4 c^{2} t^{\beta}}\right)^{i+k+1}\right.\right. \\
& \left.+\sum_{i=0}^{+\infty} \frac{(-1)^{k+\frac{n}{2}} A_{4}}{i !\left(i-k+\frac{n}{2}\right) ! \Gamma\left(1-\beta\left(i-k+\frac{n}{2}\right)-\alpha k\right)}\left(\frac{|x|^{2}}{4 c^{2} t^{\beta}}\right)^{i+\frac{n}{2}+1}\right] \\
& +\sum_{k=\frac{n}{2}}^{+\infty} \frac{\left(-a t^{\beta-\alpha}\right)^{k}}{k !}\left[\sum_{j=0}^{k-\frac{n}{2}-1} \frac{(-1)^{j}}{j !} \frac{\Gamma\left(-j+k-\frac{n}{2}\right)\left(j+\frac{n}{2}\right)}{\Gamma\left(1-\beta\left(j-k+\frac{n}{2}\right)-\alpha k\right)}\left(\frac{|x|^{2}}{4 c^{2} t^{\beta}}\right)^{j+\frac{n}{2}+1}\right. \\
& \left.\left.+\sum_{j=0}^{+\infty} \frac{(-1)^{k+\frac{n}{2}} A_{5}}{j !\left(j+k-\frac{n}{2}\right) ! \Gamma(1-\beta j-\alpha k)}\left(\frac{|x|^{2}}{4 c^{2} t^{\beta}}\right)^{j+k+1}\right]\right\}
\end{aligned}
$$

where $A_{4}$ and $A_{5}$ are the following expressions

$$
\begin{aligned}
& A_{4}=-1+\left(i+\frac{n}{2}\right)\left(\ln \left(\frac{4 c^{2} t^{\beta}}{|x|^{2}}\right)+\psi\left(1+i-k+\frac{n}{2}\right)+\psi(1+i)-\beta \psi\left(1-\beta\left(i-k+\frac{n}{2}\right)-\alpha k\right)\right) \\
& A_{5}=-1+(j+k)\left(\ln \left(\frac{4 c^{2} t^{\beta}}{|x|^{2}}\right)+\psi\left(1+j+k-\frac{n}{2}\right)+\psi(1+j)-\beta \psi(1-\beta j-\alpha k)\right)
\end{aligned}
$$

Remark 3.2 As indicated in [11], in the even case and for some rational values of $\beta$ we have an indetermination in the series coefficients due to the terms $\beta \psi\left(1-\beta\left(i-k+\frac{n}{2}\right)-\alpha k\right)$ and $\Gamma\left(1-\beta\left(i-k+\frac{n}{2}\right)-\alpha k\right)$ in the first inner series, and due to the terms $\beta \psi(1-\beta j-\alpha k)$ and $\Gamma(1-\beta j-\alpha k)$ in the second inner series. These indeterminations can be removed after applying (1) with $n=1$, (2), (3), and the relation $\psi(1-z)=$ $\pi \cot (\pi z)+\psi(z)$ for the digamma function.

\section{Fundamental solution of the time-fractional telegraph Dirac op- erator}

In this section we compute the FS for the time-fractional telegraph Dirac operator defined by

$$
D_{x, t}^{\alpha, \beta}:=c D_{x}+\mathfrak{f}\left(D_{t}^{\beta}+a D_{t}^{\alpha}\right)+\mathfrak{f}^{\dagger},
$$

where $D_{x}$ is the Dirac operator in $\mathbb{R}^{n}, a, c \in \mathbb{R}^{+}, D_{t}^{\alpha}$ and $D_{t}^{\beta}$ are the Caputo fractional derivatives of order $\alpha \in] 0,1]$ and $\beta \in] 1,2]$, respectively (see (14)). This operator factorizes the time-fractional telegraph operator $-c^{2} \Delta_{x}+D_{t}^{\beta}+a D_{t}^{\alpha}$ for Clifford valued functions $f$ given by $f=\sum_{A} e_{A} f_{A}$, where $f_{A} \in C^{2}(\Omega \times I)$, with $\Omega \times I \subseteq \mathbb{R}^{n} \times \mathbb{R}^{+}$. In fact, taking into account the multiplication rules (11) and (12) we have that

$$
\begin{aligned}
\left(D_{x, t}^{\alpha, \beta}\right)^{2}= & \left(c D_{x}+\mathfrak{f}\left(D_{t}^{\beta}+a D_{t}^{\alpha}\right)+\mathfrak{f}^{\dagger}\right)\left(c D_{x}+\mathfrak{f}\left(D_{t}^{\beta}+a D_{t}^{\alpha}\right)+\mathfrak{f}^{\dagger}\right) \\
= & c^{2} D_{x}^{2}-c \mathfrak{f} D_{x}\left(D_{t}^{\beta}+a D_{t}^{\alpha}\right)-\mathfrak{f}^{\dagger} c D_{x}+\mathfrak{f} c D_{x}\left(D_{t}^{\beta}+a D_{t}^{\alpha}\right) \\
& +\mathfrak{f}^{2}\left(D_{t}^{\beta}+a D_{t}^{\alpha}\right)\left(D_{t}^{\beta}+a D_{t}^{\alpha}\right)+\mathfrak{f f}^{\dagger}\left(D_{t}^{\beta}+a D_{t}^{\alpha}\right)+\mathfrak{f}^{\dagger} c D_{x}+\mathfrak{f}^{\dagger} \mathfrak{f}\left(D_{t}^{\beta}+a D_{t}^{\alpha}\right)+\left(\mathfrak{f}^{\dagger}\right)^{2} \\
= & c^{2} D_{x}^{2}+\left(\mathfrak{f} \mathfrak{f}^{\dagger}+\mathfrak{f}^{\dagger} \mathfrak{f}\right)\left(D_{t}^{\beta}+a D_{t}^{\alpha}\right) \\
= & -c^{2} \Delta_{x}+D_{t}^{\beta}+a D_{t}^{\alpha} .
\end{aligned}
$$

The FS of the time-fractional telegraph Dirac operator will be denoted by $\mathfrak{G}_{n}^{\alpha, \beta}$ and is obtained by the application of the operator $D_{x, t}^{\alpha, \beta}$ to $G_{n}^{\alpha, \beta}$, i.e.,

$$
\mathfrak{G}_{n}^{\alpha, \beta}(x, t)=D_{x, t}^{\alpha, \beta} G_{n}^{\alpha, \beta}(x, t)=c D_{x} G_{n}^{\alpha, \beta}(x, t)+\mathfrak{f}\left(D_{t}^{\beta}+a D_{t}^{\alpha}\right) G_{n}^{\alpha, \beta}(x, t)+\mathfrak{f}^{\dagger} G_{n}^{\alpha, \beta}(x, t) .
$$

Indeed, by the above factorization we have $D_{x, t}^{\alpha, \beta} G_{n}^{\alpha, \beta}(x, t)=\left(-c^{2} \Delta_{x}+D_{t}^{\beta}+a D_{t}^{\alpha}\right) G_{n}^{\alpha, \beta}(x, t)=0$, which shows that $\mathfrak{G}_{n}^{\alpha, \beta}$ is the FS of $D_{x, t}^{\alpha, \beta}$. First, we are going to deduce an integral representation for $\mathfrak{G}_{n}^{\alpha, \beta}$. Taking into 
account the series representation of the Bessel function and the following relation proved in [8]

$$
D_{x}\left[|x|^{k}\right]=\sum_{i=1}^{n} e_{i} \partial_{x_{i}}\left(\left(\sum_{j=1}^{n} x_{j}^{2}\right)^{\frac{k}{2}}\right)=k|x|^{k-2} x, \quad k \in \mathbb{N}_{0},
$$

we obtain

$$
D_{x}\left[|x|^{1-\frac{n}{2}} J_{\frac{n}{2}-1}(\tau|x|)\right]=-\frac{\tau x}{|x|^{\frac{n}{2}}} J_{\frac{n}{2}}(\tau|x|) .
$$

Moreover, taking into account (14), together with the series expansion of the multivariate Mittag-Leffler function (5), we have

$$
\begin{aligned}
& D_{t}^{\beta}\left[t^{\beta} E_{(\beta-\alpha, \beta), 1+\beta}\left(-a t^{\beta-\alpha},-c^{2} t^{\beta}\right)\right]=E_{(\beta-\alpha, \beta), 1}\left(-a t^{\beta-\alpha},-c^{2} t^{\beta}\right), \\
& D_{t}^{\alpha}\left[t^{\beta} E_{(\beta-\alpha, \beta), 1+\beta}\left(-a t^{\beta-\alpha},-c^{2} t^{\beta}\right)\right]=t^{\beta-\alpha} E_{(\beta-\alpha, \beta), 1+\beta-\alpha}\left(-a t^{\beta-\alpha},-c^{2} t^{\beta}\right) .
\end{aligned}
$$

Thereby, applying $D_{x, t}^{\alpha, \beta}$ to (18), and using the Leibniz rule for improper integrals together with relations (22), (23), and (24), we get

$$
\begin{aligned}
\mathfrak{G}_{n}^{\alpha, \beta}(x, t)= & \left(c D_{x}+\mathfrak{f}^{\dagger}\right) \delta(x)-\frac{c^{3} t^{\beta} x}{(2 \pi)^{\frac{n}{2}}|x|^{\frac{n}{2}}} \int_{0}^{+\infty} \tau^{\frac{n}{2}+3} E_{(\beta-\alpha, \beta), 1+\beta}\left(-a t^{\beta-\alpha},-c^{2} t^{\beta}\right) J_{\frac{n}{2}}(\tau|x|) d \tau \\
& -\mathfrak{f} \frac{c^{2}|x|^{1-\frac{n}{2}}}{(2 \pi)^{\frac{n}{2}}} \int_{0}^{+\infty} \tau^{\frac{n}{2}+2}\left[E_{(\beta-\alpha, \beta), 1}\left(-a t^{\beta-\alpha},-c^{2} t^{\beta}\right)\right. \\
& \left.\quad+a t^{\beta-\alpha} E_{(\beta-\alpha, \beta), 1+\beta-\alpha}\left(-a t^{\beta-\alpha},-c^{2} t^{\beta}\right) J_{\frac{n}{2}-1}(\tau|x|) d \tau\right] \\
& -\mathfrak{f}^{\dagger} \frac{c^{2}|x|^{1-\frac{n}{2}} t^{\beta}}{(2 \pi)^{\frac{n}{2}}} \int_{0}^{+\infty} \tau^{\frac{n}{2}+2} E_{(\beta-\alpha, \beta), 1+\beta}\left(-a t^{\beta-\alpha},-c^{2} t^{\beta}\right) J_{\frac{n}{2}-1}(\tau|x|) d \tau .
\end{aligned}
$$

Applying $D_{x, t}^{\alpha, \beta}$ to (19) and taking into account Corollary 2.5 it is possible to write the components of $\mathfrak{G}_{n}^{\alpha, \beta}$ as H-functions of two variables.

Theorem 4.1 The FS has the following representation:

$$
\begin{aligned}
& \mathfrak{G}_{n}^{\alpha, \beta}(x, t)=\left(c D_{x}+\mathfrak{f}^{\dagger}\right) \delta(x)+\frac{8 c^{3} t^{\beta} x}{\pi^{\frac{n}{2}}|x|^{n+4}} H_{1,1 ; 0,1 ; 2,1}^{0,1 ; 1,0 ; 1,1}\left[\begin{array}{c|c}
a t^{\beta-\alpha} & (0 ; 1,1) ;-;\left(-1-\frac{n}{2}, 1\right),(-1,1) \\
4 c^{2}|x|^{-2} t^{\beta} & (-\beta ; \beta-\alpha, \beta) ;(0,1) ;(0,1)
\end{array}\right] \\
& -\mathfrak{f} \frac{4 c^{2}}{\pi^{\frac{n}{2}}|x|^{n+2}}\left(\begin{array}{c|c}
H_{1,1 ; 0,1 ; 2,1}^{0,1 ; 1,0 ; 1,1}\left[\begin{array}{c}
a t^{\beta-\alpha} \\
4 c^{2}|x|^{-2} t^{\beta}
\end{array}\right. & (0 ; 1,1) ;-;\left(-\frac{n}{2}, 1\right),(-1,1) \\
(0 ; \beta-\alpha, \beta) ;(0,1) ;(0,1)
\end{array}\right] \\
& \left.+H_{1,1 ; 0,1 ; 1,1}^{0,1 ; 1,0 ; 1,1}\left[\begin{array}{c|c}
a t^{\beta-\alpha} & (0 ; 1,1) ;-;\left(-\frac{n}{2}, 1\right) \\
4 c^{2}|x|^{-2} t^{\beta} & (-(\beta-\alpha) ; \beta-\alpha, \beta) ;(0,1) ;(0,1)
\end{array}\right]\right) \\
& -\mathfrak{f}^{+} \frac{4 c^{2} t^{\beta}}{\pi^{\frac{n}{2}}|x|^{n+2}} H_{1,1 ; 0,1 ; 2,1}^{0,1 ; 1,0 ; 1,1}\left[\begin{array}{c|c}
a t^{\beta-\alpha} & (0 ; 1,1) ;-;\left(-\frac{n}{2}, 1\right),(-1,1) \\
4 c^{2}|x|^{-2} t^{\beta} & (-\beta ; \beta-\alpha, \beta) ;(0,1) ;(0,1)
\end{array}\right] .
\end{aligned}
$$

It's not difficult to see that the H-function of two variables defined in (26) are convergent for all $0<\alpha<1$ and $1<\beta \leq 2$. Indeed, applying the conditions (6) and (7) we have that a $a t^{\beta-\alpha}$ and $4 c^{2}|x|^{-2} t^{\beta}$ are non-negative real numbers, which implies that their argument is always zero and $\Omega_{1}=2+\alpha-\beta>0$ for all the H-functions in (26), and $\Omega_{2}=2-\beta \geq 0$ for the 1 st, 2 nd and 4 th, while $\Omega_{2}=3-\beta>0$ for the 3rd. This proves the convergence of the double Mellin-Barnes integrals that represent the H-functions of two varibales in (26). In order to get a series representation for $\mathfrak{G}_{n}^{\alpha, \beta}$ we apply $D_{x, t}^{\alpha, \beta}$ to (20) and (21). The following formula concerning the Dirac operator is necessary to compute $\mathfrak{G}_{n}^{\alpha, \beta}$ (see [8]):

$$
D_{x}\left[\left(C+\ln \left(\frac{4 c^{2} t^{\beta}}{|x|^{2}}\right)\right)|x|^{2 k}\right]=2 x|x|^{2 k-2}\left(k\left(C+\ln \left(\frac{4 c^{2} t^{\beta}}{|x|^{2}}\right)\right)-1\right), \quad C \in \mathbb{R} .
$$


Concerning the Caputo fractional derivative we use the symbolic calculus software Mathematica. When $0<$ $\alpha \leq 1$ we obtain

$$
\begin{aligned}
& D_{t}^{\alpha}\left[\left(C+\ln \left(\frac{4 c^{2} t^{\beta}}{|x|^{2}}\right)\right) t^{-\beta\left(k+\frac{n}{2}\right)}\right] \\
& =\frac{\Gamma\left(1-\beta\left(k+\frac{n}{2}\right)\right)}{t^{\beta\left(k+\frac{n}{2}\right)+\alpha} \Gamma\left(1-\beta\left(k+\frac{n}{2}\right)-\alpha\right)} \times\left[C+\beta \pi \cot \left(\beta \pi\left(k+\frac{n}{2}\right)\right)-\beta H\left(1-\beta\left(k+\frac{n}{2}\right)\right)\right. \\
& \left.\quad-\beta H\left(-\alpha+\beta\left(k+\frac{n}{2}\right)\right)+\ln \left(\frac{4 c^{2} t^{\beta}}{|x|^{2}}\right)\right] \\
& =\frac{\Gamma\left(1-\beta\left(k+\frac{n}{2}\right)\right)}{t^{\beta\left(k+\frac{n}{2}\right)+\alpha} \Gamma\left(1-\beta\left(k+\frac{n}{2}\right)-\alpha\right)} \times\left[C+\beta \psi\left(1-\beta\left(k+\frac{n}{2}\right)\right)-\beta \psi\left(1-\beta\left(k+\frac{n}{2}\right)-\alpha\right)+\ln \left(\frac{4 c^{2} t^{\beta}}{|x|^{2}}\right)\right]
\end{aligned}
$$

with $C \in \mathbb{R}$. In (27) $H$ denotes the generalized Harmonic number. To obtain (28) we used the identities $H(z)=\psi(z+1)+\gamma$ and $\psi(1-z)=\pi \cot (\pi z)+\psi(z)$, where $\gamma$ is the Euler-Mascheroni constant. When $1<\beta \leq 2$ we obtain (see [8])

$$
\begin{aligned}
& D_{t}^{\beta}\left[\left(C+\ln \left(\frac{4 c^{2} t^{\beta}}{|x|^{2}}\right)\right) t^{-\beta\left(k+\frac{n}{2}\right)}\right] \\
& =\frac{\Gamma\left(1-\beta\left(k+\frac{n}{2}\right)\right)}{t^{\beta\left(k+\frac{n}{2}+1\right)} \Gamma\left(1-\beta\left(k+\frac{n}{2}+1\right)\right)} \times\left[C+\beta \psi\left(1-\beta\left(k+\frac{n}{2}\right)\right)-\beta \psi\left(1-\beta\left(k+\frac{n}{2}+1\right)\right)+\ln \left(\frac{4 c^{2} t^{\beta}}{|x|^{2}}\right)\right],
\end{aligned}
$$

with $C \in \mathbb{R}$. Finally, applying $D_{x, t}^{\alpha, \beta}$ to $(20)$ and $(21)$ and after straightforward calculations we obtain the following theorems.

Theorem 4.2 For $n$ odd, $0<\alpha \leq 1$, and $1<\beta \leq 2$ the FS of the time-fractional telegraph Dirac operator is given by

$$
\begin{aligned}
& \mathfrak{G}_{n}^{\alpha, \beta}(x, t)=\left(c D_{x}+\mathfrak{f}^{\dagger}\right) \delta(x)+\frac{c x}{\pi^{\frac{n}{2}}|x|^{2}} \sum_{k=0}^{+\infty} \frac{\left(-a t^{\beta-\alpha}\right)^{k}}{k !}\left[\sum_{q=0}^{\frac{n-2 k-1}{2}} \frac{2(-1)^{q} \Gamma\left(\frac{n}{2}-q-k+1\right)(q+k)}{|x|^{n} \Gamma(1-\beta q+\alpha k) q !}\left(\frac{|x|^{2}}{4 c^{2} t^{\beta}}\right)^{q+k}\right. \\
& \left.-\frac{(-1)^{\frac{n-1}{2}} \sqrt{\pi}|x|}{2^{n}\left(c t^{\frac{\beta}{2}}\right)^{n+1}} \sum_{p=0}^{+\infty} \frac{\left(-\frac{p+1+n}{2}\right)_{k}}{\left(\frac{p+2}{2}\right)_{\frac{n-1}{2}} \Gamma\left(1-\frac{\beta(p+1+n)}{2}+(\beta-\alpha) k\right) p !}\left(\frac{-|x|}{c t^{\frac{\beta}{2}}}\right)^{p}\right] \\
& -\mathfrak{f} \frac{1}{\pi^{\frac{n}{2}}|x|^{n}} \sum_{k=0}^{+\infty} \frac{\left(-a t^{\beta-\alpha}\right)^{k}}{k !}\left[\sum_{q=0}^{\frac{n-2 k-1}{2}} \frac{(-1)^{q} \Gamma\left(\frac{n}{2}-q-k\right)(q+k)}{q !} \frac{1-\beta(q+1)-\alpha k+a t^{\beta}}{t^{\beta} \Gamma(2-\beta(q+1)-\alpha k)}\left(\frac{|x|^{2}}{4 c^{2} t^{\beta}}\right)^{q+k}\right. \\
& \left.-\frac{(-1)^{\frac{n-1}{2}} \sqrt{\pi}}{2^{n} t^{\beta+k}}\left(\frac{|x|^{2}}{c^{2} t^{\beta}}\right)^{\frac{n}{2}} \sum_{p=0}^{+\infty} \frac{\left(-\frac{p+n}{2}\right)_{k}}{\left(\frac{p+1}{2}\right)_{\frac{n-1}{2}} p !} \frac{1-\frac{\beta(p+n+2)}{2}+(\beta-\alpha) k+a t}{\Gamma\left(2-\frac{\beta(p+n+2)}{2}+(\beta-\alpha) k\right)}\left(\frac{-|x|}{c t^{\frac{\beta}{2}}}\right)^{p}\right] \\
& -\mathfrak{f}^{\dagger} \frac{1}{\pi^{\frac{n}{2}}|x|^{n}} \sum_{k=0}^{+\infty} \frac{\left(-a t^{\beta-\alpha}\right)^{k}}{k !}\left[\sum_{q=0}^{\frac{n-2 k-1}{2}} \frac{(-1)^{q} \Gamma\left(\frac{n}{2}-q-k\right)(q+k)}{\Gamma(1-\beta q-\alpha k) q !}\left(\frac{|x|^{2}}{4 c^{2} t^{\beta}}\right)^{q+k}\right. \\
& \left.+\frac{(-1)^{\frac{n-1}{2}} \sqrt{\pi}|x|^{n}}{\left(2 c t^{\frac{\beta}{2}}\right)^{n}} \sum_{p=0}^{+\infty} \frac{\left(-\frac{p+n}{2}\right)_{k}}{\left(\frac{p+1}{2}\right)_{\frac{n-1}{2}} \Gamma\left(1-\frac{\beta(p+n)}{2}+(\beta-\alpha) k\right)}\left(-\frac{|x|}{c t^{\frac{\beta}{2}}}\right)^{p}\right] \text {. }
\end{aligned}
$$

From (8) we conclude that in the odd case the function $\mathfrak{G}_{n}^{\alpha, \beta}$ has the following asymptotic behaviour at infinity

$$
\mathfrak{G}_{n}^{\alpha, \beta}(x, t)=x O\left(t^{-\frac{\beta n}{2}-\beta}\right)+\mathfrak{f} O\left(t^{-\frac{\beta n}{2}-\beta}\right)+\mathfrak{f}^{\dagger} O\left(t^{-\frac{\beta n}{2}}\right) .
$$

Remark 4.3 When $n=1, a=c=1, \beta=1$ and $\alpha=\frac{1}{2}$, our time-fractional telegraph Dirac operator factorizes the heat operator with a damping term (for more details see [20]). For this particular case we have the following 
series representation of $\mathfrak{G}_{1}^{\frac{1}{2}, 1}$

$$
\begin{aligned}
\mathfrak{G}_{1}^{\frac{1}{2}, 1}(x, t) & =\left(D_{x}+\mathfrak{f}^{\dagger}\right) \delta(x)-\frac{x}{2 t|x|} \sum_{k=0}^{+\infty} \frac{(-\sqrt{t})^{k}}{k !} \sum_{p=0}^{+\infty} \frac{\left(-\frac{p+2}{2}\right)_{k}}{\Gamma\left(\frac{k-p}{2}\right) p !}\left(-\frac{|x|}{\sqrt{t}}\right)^{p} \\
& +\mathfrak{f} \frac{1}{2 \sqrt{t^{3}}} \sum_{k=0}^{+\infty} \frac{(-1)^{k}}{\sqrt{t^{k}} k !} \sum_{p=0}^{+\infty} \frac{\left(-\frac{p+1}{2}\right)_{k}\left(\frac{k-p-1}{2}+t\right)}{\Gamma\left(\frac{k-p+1}{2}\right) p !}\left(-\frac{|x|}{\sqrt{t}}\right)^{p} \\
& +\mathfrak{f}^{\dagger} \frac{1}{2 \sqrt{t}} \sum_{k=0}^{+\infty} \frac{(-\sqrt{t})^{k}}{k !} \sum_{p=0}^{+\infty} \frac{\left(-\frac{p+1}{2}\right)_{k}}{\Gamma\left(\frac{k-p+1}{2}\right) p !}\left(-\frac{|x|}{t^{\frac{\beta}{2}}}\right)^{p}
\end{aligned}
$$

Theorem 4.4 For $n$ even, $0<\alpha \leq 1$, and $1<\beta \leq 2$ the vectorial component of the FS of the time-fractional telegraph Dirac operator is given by

$$
\begin{aligned}
& c D_{x} G_{n}^{\alpha, \beta}(x, t)=c D_{x} \delta(x)-\frac{8 c^{3} t^{\beta} x}{\pi^{\frac{n}{2}}|x|^{n+1}}\left[\sum _ { k = 0 } ^ { \frac { n } { 2 } - 1 } \frac { ( - a t ^ { \beta - \alpha } ) ^ { k } } { k ! } \left[\sum_{i=0}^{\frac{n}{2}-k-1} \frac{(-1)^{i+1}}{i !} \frac{\Gamma\left(1-i-k+\frac{n}{2}\right)}{\Gamma(1-\beta i-\alpha k)}\left(\frac{|x|^{2}}{4 c^{2} t^{\beta}}\right)^{i+k+1}\right.\right. \\
& \left.+\sum_{p=0}^{\infty} \frac{(-1)^{k+\frac{n}{2}}\left(p\left(A_{1}+\left(p+\frac{n}{2}\right) \ln \left(\frac{4 c^{2} t^{\beta}}{|x|^{2}}\right)\right)-\left(p+\frac{n}{2}\right)\right)}{\left(p-k-\frac{n}{2}\right) ! p ! \Gamma\left(1-\beta\left(p-k+\frac{n}{2}\right)-\alpha k\right)}\left(\frac{|x|^{2}}{4 c^{2} t^{\beta}}\right)^{1+p+\frac{n}{2}}\right] \\
& +\sum_{k=\frac{n}{2}}^{\infty} \frac{\left(-a t^{\beta-\alpha}\right)^{k}}{k !}\left[\sum_{j=1}^{k-\frac{n}{2}-1} \frac{(-1)^{j}}{(j-1) !} \frac{\Gamma\left(-j+k-\frac{n}{2}\right)\left(j+\frac{n}{2}\right)}{\Gamma\left(1-\beta\left(j-k+\frac{n}{2}\right)-\alpha k\right)}\left(\frac{|x|^{2}}{4 c^{2} t^{\beta}}\right)^{1+j+\frac{n}{2}}\right. \\
& \left.\left.+\sum_{q=0}^{+\infty} \frac{(-1)^{k+\frac{n}{2}}\left(\left(q+k-\frac{n}{2}\right)\left(A_{2}+(q+k) \ln \left(\frac{4 c^{2} t^{\beta}}{|x|^{2}}\right)\right)-(q+k)\right)}{\left(q+k-\frac{n}{2}\right) ! q ! \Gamma(1-\alpha k-\beta q)}\left(\frac{|x|^{2}}{4 c^{2} t^{\beta}}\right)^{1+q+\frac{n}{2}}\right]\right]
\end{aligned}
$$

The $\mathfrak{f}$-component is given by

$$
\begin{aligned}
\mathfrak{f}\left(D_{t}^{\beta}\right. & \left.+a D_{t}^{\alpha}\right) G_{n}^{\alpha, \beta}(x, t)=-\mathfrak{f} \frac{4 c^{2}}{\pi^{\frac{n}{2}}|x|^{n+2}}\left\{\sum _ { k = 0 } ^ { \frac { n } { 2 } - 1 } \frac { ( - a t ^ { \beta - \alpha } ) ^ { k } } { k ! } \left[\sum_{i=0}^{\frac{n}{2}-k-1} \frac{(-1)^{i} \Gamma\left(-i-k+\frac{n}{2}\right)(i+k)}{t^{\beta(i+k)} i !}\right.\right. \\
& \times \frac{\Gamma(1-\beta i-\alpha(k+1)) t^{-\beta}+\Gamma(1-\beta(i+1)-\alpha k) a t^{-\alpha}}{\Gamma(1-\beta(i+1)-\alpha k) \Gamma(1-\beta i-\alpha(k+1))}\left(\frac{4 c^{2}}{|x|^{2}}\right)^{-i-k-1} \\
& +\sum_{p=0}^{+\infty} \frac{(-1)^{k+\frac{n}{2}}\left(p+\frac{n}{2}\right)}{t^{\beta\left(p+\frac{n}{2}\right)}\left(p+\frac{n}{2}-k\right) ! \Gamma\left(1-\beta\left(p+\frac{n}{2}+1\right)+\beta\left(1-\frac{\alpha}{\beta}\right) k\right) p !}\left(\frac{4 c^{2}}{|x|^{2}}\right)^{-1-p-\frac{n}{2}} \\
& \times\left[(1+a)\left[\frac{A_{1}}{p+\frac{n}{2}}+\beta \psi\left(1-\beta\left(p+\frac{n}{2}\right)+\beta\left(1-\frac{\alpha}{\beta}\right) k\right)+\ln \left(\frac{4 c^{2} t^{\beta}}{|x|^{2}}\right)\right]\right. \\
& \left.\left.-\beta \psi\left(1-\beta\left(p+\frac{n}{2}+1\right)+\beta\left(1-\frac{\alpha}{\beta}\right) k\right)-a \beta \psi\left(1-\alpha-\beta\left(p+\frac{n}{2}\right)+\beta\left(1-\frac{\alpha}{\beta}\right) k\right)\right]\right] \\
+ & \sum_{k=\frac{n}{2}}^{\infty} \frac{\left(-a t^{\beta-\alpha}\right)^{k}}{k !}\left[\sum_{j=0}^{k-\frac{n}{2}-1} \frac{(-1)^{j} \Gamma\left(-j+k-\frac{n}{2}\right)}{t^{\beta\left(j+\frac{n}{2}\right)} j !}\left(\frac{4 c^{2}}{|x|^{2}}\right)^{-1-j-\frac{n}{2}}\right. \\
& \times \frac{\Gamma\left(1-\alpha-\beta\left(j+\frac{n}{2}\right)+(\beta-\alpha) k\right) t^{-\beta}+\Gamma\left(1-\beta\left(j+\frac{n}{2}+1\right)+(\beta-\alpha) k\right) a t^{-\alpha}}{\Gamma\left(1-\beta\left(j+\frac{n}{2}+1\right)+(\beta-\alpha) k\right) \Gamma\left(1-\alpha-\beta\left(j+\frac{n}{2}\right)+(\beta-\alpha) k\right)} \\
+ & \sum_{q=0}^{+\infty} \frac{(-1)^{k+\frac{n}{2}}(q+k) t^{-\beta(q+k+1)}}{\left(q+k+\frac{n}{2}\right) ! q ! \Gamma(1-\beta(q+1)-\alpha k)}\left(\frac{4 c^{2}}{|x|^{2}}\right)^{-1-q-k}\left[( 1 + a ) \left[\frac{A_{2}}{q+k}+\beta \psi(1+\beta q-\alpha k)\right.\right. \\
& \left.\left.\left.\left.+\ln \left(\frac{4 c^{2} t^{\beta}}{|x|^{2}}\right)\right]-\beta \psi(1-\beta(q+1)-\alpha k)-a \beta \psi(1-\beta q-\alpha(k+1))\right]\right]\right\} .
\end{aligned}
$$


In the previous expression $A_{1}$ and $A_{2}$ are given by

$$
\begin{aligned}
& A_{1}=-1+\left(p+\frac{n}{2}\right)\left[\psi\left(1+p+\frac{n}{2}-k\right)-\beta \psi\left(1-\beta\left(p+\frac{n}{2}\right)+\beta\left(1-\frac{\alpha}{\beta}\right) k\right)+\psi(1+p)\right], \\
& A_{2}=-1+(q+k)\left[\psi\left(1+q+k-\frac{n}{2}\right)-\beta \psi(1-\alpha k-\beta q)+\psi(1+q)\right] .
\end{aligned}
$$

The $\mathfrak{f}^{\dagger}$-component is given by (21) multiplied by $\mathfrak{f}^{\dagger}$.

Remark 4.5 If we consider $a=0$ in (29), (31), (32) and (21), which implies that the only non-null term in the outer series is the first one, we get the expressions deduced in [8, Sec. 4] for the FS of the time-fractional parabolic Dirac operator.

\section{Graphical representations of $\mathfrak{G}_{n}^{\alpha, \beta}$}

In this section we present and discuss some plots of $\mathfrak{G}_{n}^{\alpha, \beta}$, for $a, c=1, n=1,3$ and some values of the fractional parameters $\alpha$ and $\beta$, using the series representations described in Theorem 4.2. Due to the complexity of the series representation for the even case we only present plots for the odd case. Let us remark that in some cases these series are not suitable to numerically evaluate the solution as soon as $|x|$ is sufficiently small or sufficiently large, due to the presence of positive and negative powers of $|x|$. The plots were made summing the alternate series using the MatLab code ALTSUM [19], which provides a convergence acceleration of alternating series.

\subsection{Case $n=1$}

Considering $n=a=c=1$ in (29) we obtain the following expression for $\mathfrak{G}_{1}^{\alpha, \beta}$ :

$$
\begin{aligned}
\mathfrak{G}_{1}^{\alpha, \beta}(x, t) & =\left(D_{x}+\mathfrak{f}^{\dagger}\right) \delta(x)-\frac{x}{2 t^{\beta}|x|} \sum_{k=0}^{+\infty} \frac{\left(-t^{\beta-\alpha}\right)^{k}}{k !} \sum_{p=0}^{+\infty} \frac{\left(-\frac{p+2}{2}\right)_{k}}{\Gamma\left(1-\frac{\beta(p+2)}{2}+(\beta-\alpha) k\right) p !}\left(-\frac{|x|}{t^{\frac{\beta}{2}}}\right)^{p} \\
& +\mathfrak{f} \frac{1}{2 t^{\frac{3 \beta}{2}}} \sum_{k=0}^{+\infty} \frac{\left(-t^{\beta-\alpha}\right)^{k}}{t^{k} k !} \sum_{p=0}^{+\infty} \frac{\left(-\frac{p+1}{2}\right)_{k}\left(1-\frac{\beta(p+3)}{2}+(\beta-\alpha) k+t\right)}{\Gamma\left(2-\frac{\beta(p+3)}{2}+(\beta-\alpha) k\right) p !}\left(-\frac{|x|}{t^{\frac{\beta}{2}}}\right)^{p} \\
& +\mathfrak{f}^{\dagger} \frac{1}{2 t^{\frac{\beta}{2}}} \sum_{k=0}^{+\infty} \frac{\left(-t^{\beta-\alpha}\right)^{k}}{k !} \sum_{p=0}^{+\infty} \frac{\left(-\frac{p+1}{2}\right)_{k}}{\Gamma\left(1-\frac{\beta(p+1)}{2}+(\beta-\alpha) k\right) p !}\left(-\frac{|x|}{t^{\frac{\beta}{2}}}\right)^{p}
\end{aligned}
$$

In Figures 1, 2 and 3 we show some plots of the real, $\mathfrak{f}$ and $\mathfrak{f}^{\dagger}$-components of the reduced Green function $\mathfrak{G}_{1}^{\alpha, \beta}(x, 1)$ for some values of $\alpha$ and $\beta$.
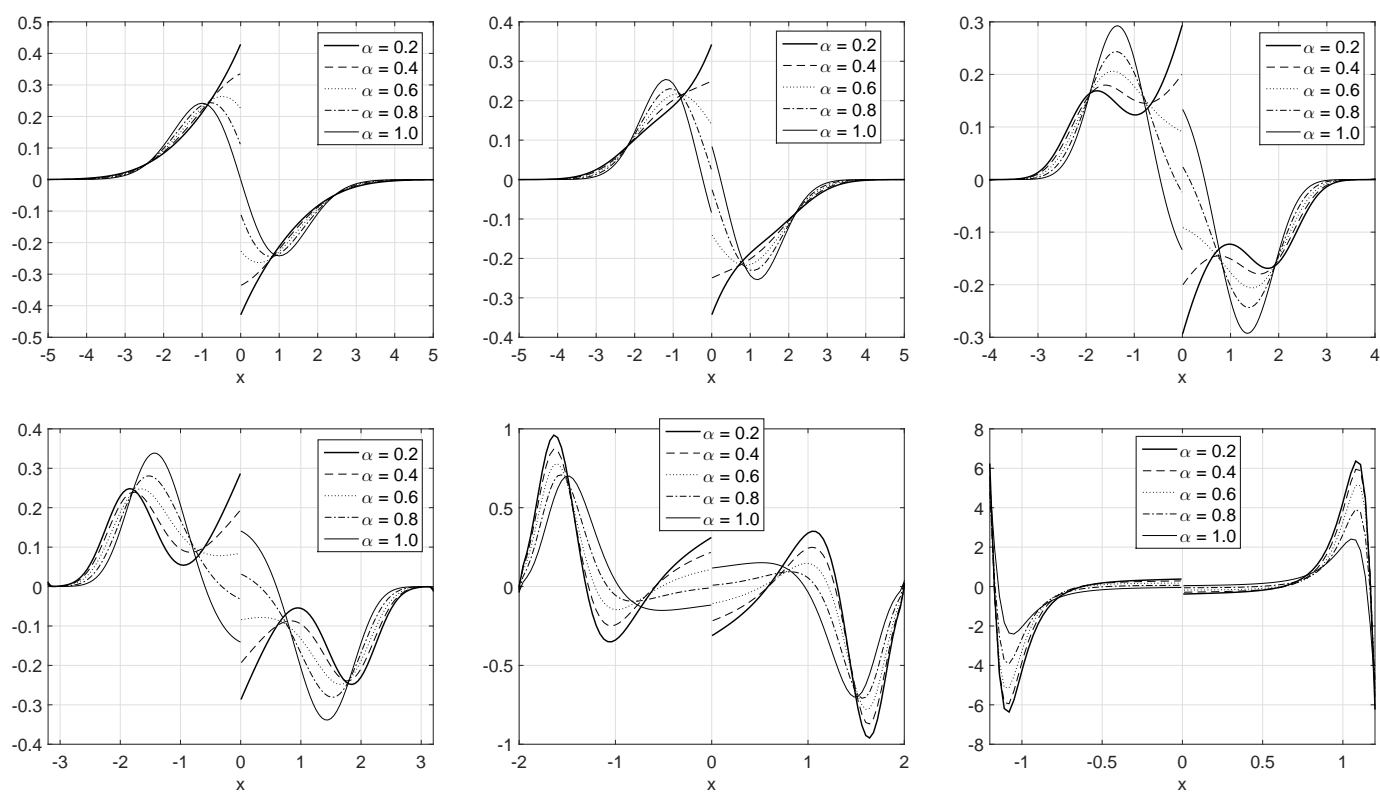

Figure 1: Plots of the real-component of $\mathfrak{G}_{1}^{\alpha, \beta}(x, 1)$ for $\alpha=0.2,0.4,0.6,0.8,1.0, \beta=1.0,1.2,1.4$ (1st line, from left to right), and $\beta=1.5,1.7,1.9$ (2nd line, from left to right). 

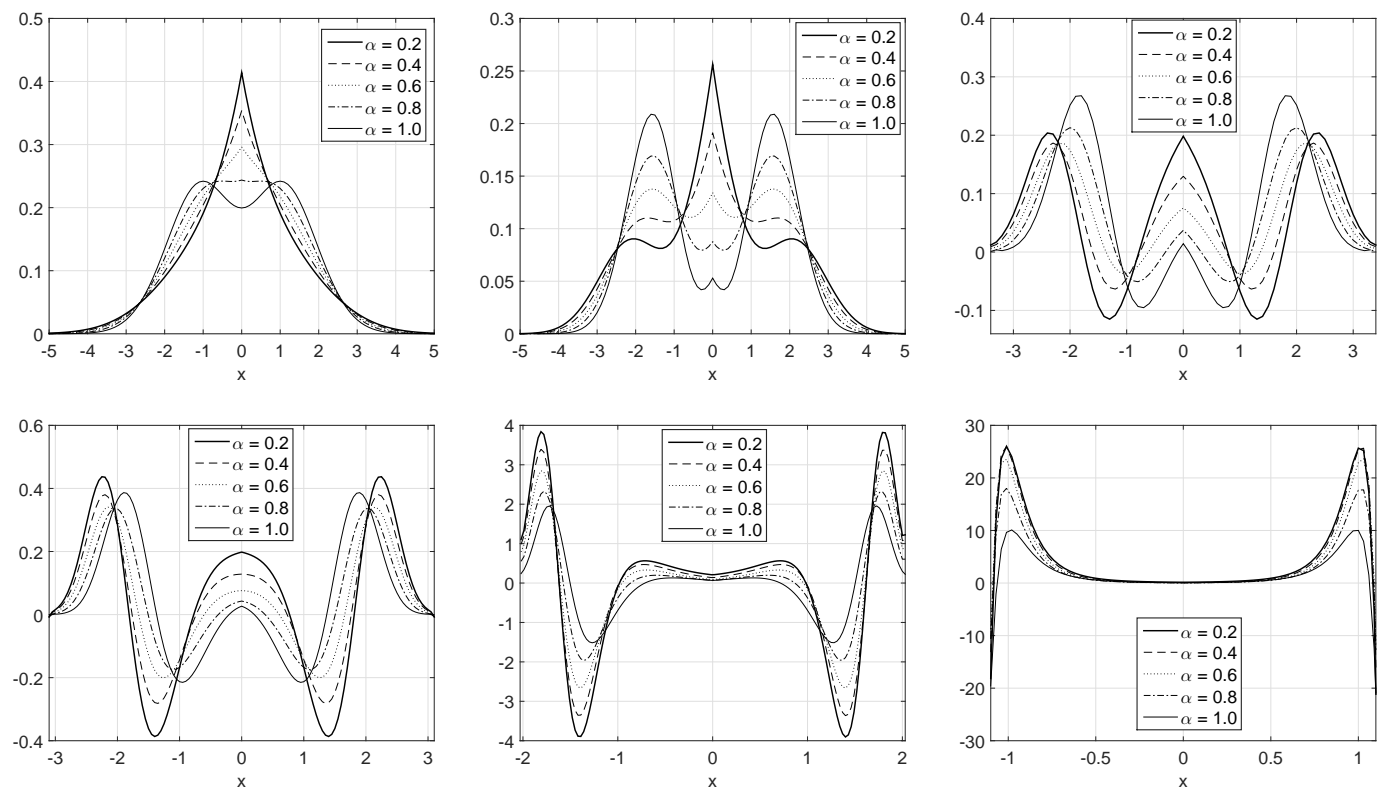

Figure 2: Plots of the $\mathfrak{f}$-component of $\mathfrak{G}_{1}^{\alpha, \beta}(x, 1)$ for $\alpha=0.2,0.4,0.60 .81 .0, \beta=1.0,1.2,1.4$ (1st line, from left to right), and $\beta=1.5,1.7,1.9$ (2nd line, from left to right).
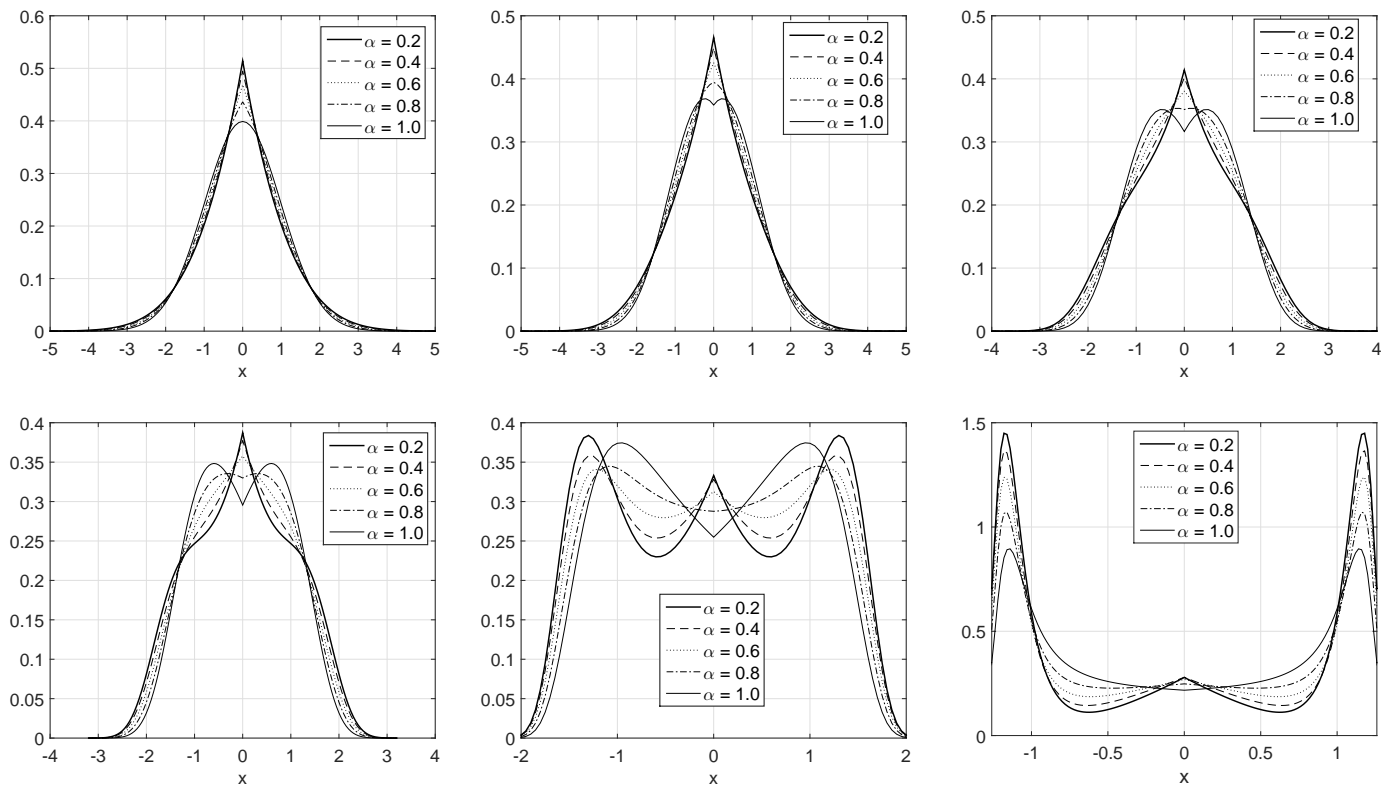

Figure 3: Plots of the $\mathfrak{f}^{\dagger}$-component of $\mathfrak{G}_{1}^{\alpha, \beta}(x, 1)$ for $\alpha=0.2,0.4,0.6,0.8,1.0, \beta=1.0,1.2,1.4$ (1st line, from left to right), and $\beta=1.5,1.7,1.9$ (2nd line, from left to right).

From the plots of Figures 1, 2 and 3 we see that the real-component is an odd function and discontinuous at $x=0$ (except when $\beta=1$ ), while the $\mathfrak{f}$ and $\mathfrak{f}^{\dagger}$-components are even continuous functions. As a consequence of the perturbations due to the parameter $\alpha$, these plots are deformations of those presented in Section 8.1 of [8]. In all the components we see that the number of extreme points increases and move apart from the origin with $\beta$. In the real component the extreme points are symmetric with respect to the origin, while in the $\mathfrak{f}$ and $\mathfrak{f}^{\dagger}$-components they are symmetric with respect to the $y$-axis. Moreover, the plots shrink horizontally as $\beta \rightarrow 2^{-}$. The influence of the parameter $\alpha$ can also be seen in the absolute value of the extreme points, since the increase of $\alpha$ results in the increase of the absolute value of the extreme points.

In Figures 4, 5, and 6 we show the time evolution of each component of $\mathfrak{G}_{1}^{\alpha, \beta}$, for $a, c=1$ and some values of $\alpha$ and $\beta$. 

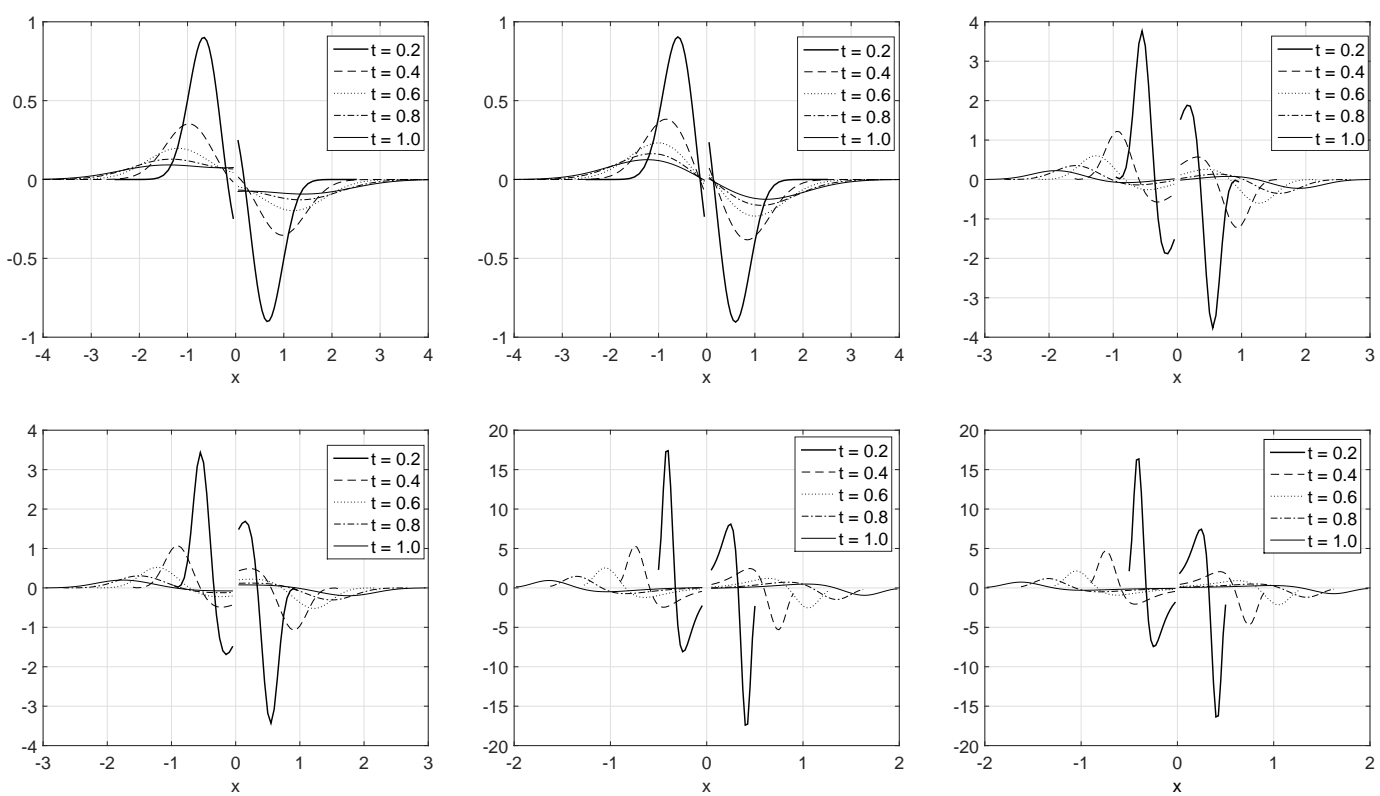

Figure 4: Plots of the real-component of $\mathfrak{G}_{1}^{\alpha, \beta}$ for the following pairs of fractional parameters $(\alpha, \beta):(0.2,1.2)$ $(0.6,1.2),(0.2,1.5)$ (1st line, from left to right), $(0.6,1.5),(0.2,1.7),(0.6,1.7)$ (2nd line, from left to right).
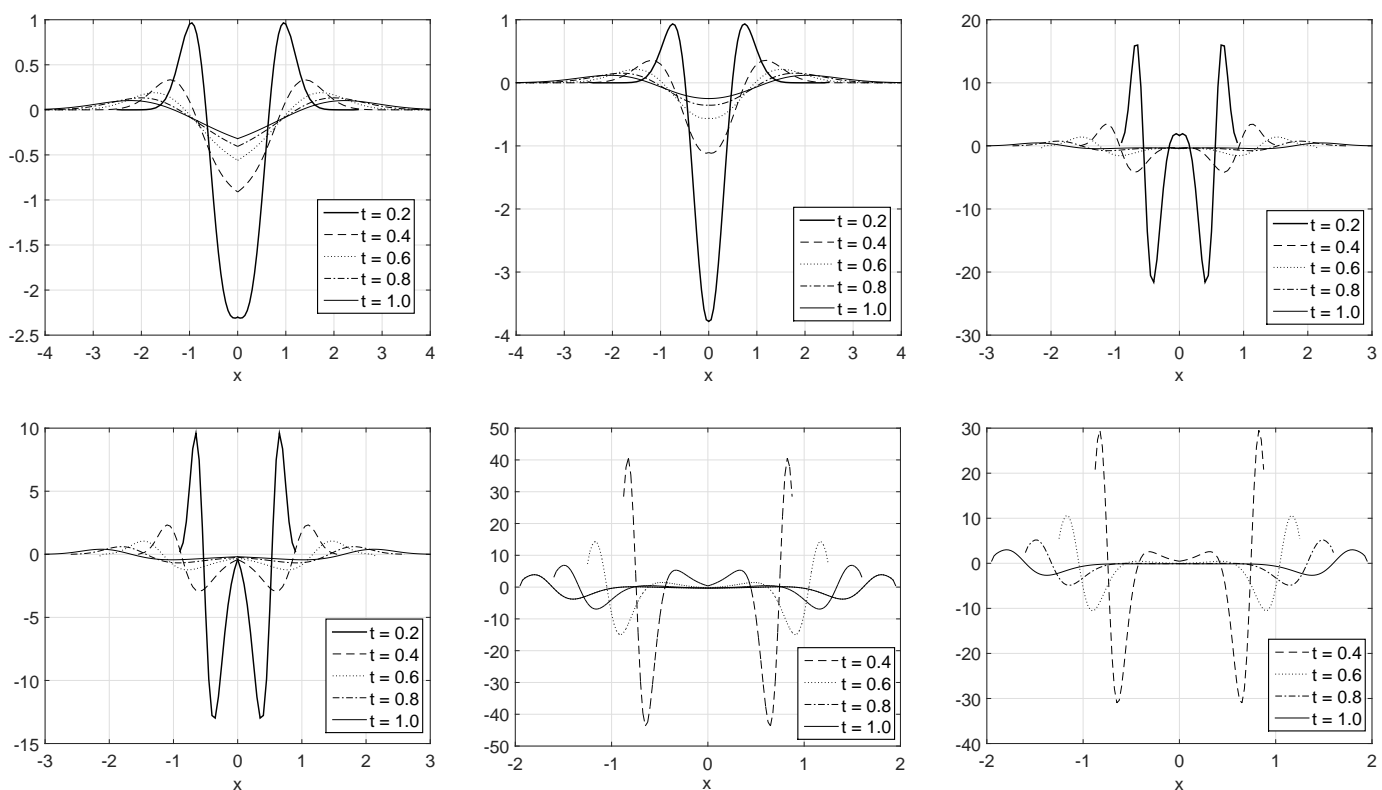

Figure 5: Plots of the $\mathfrak{f}$-component of $\mathfrak{G}_{1}^{\alpha, \beta}$ for the following pairs of fractional parameters $(\alpha, \beta):(0.2,1.2)$ $(0.6,1.2),(0.2,1.5)$ (1st line, from left to right), $(0.6,1.5),(0.2,1.7),(0.6,1.7)$ (2nd line, from left to right). 

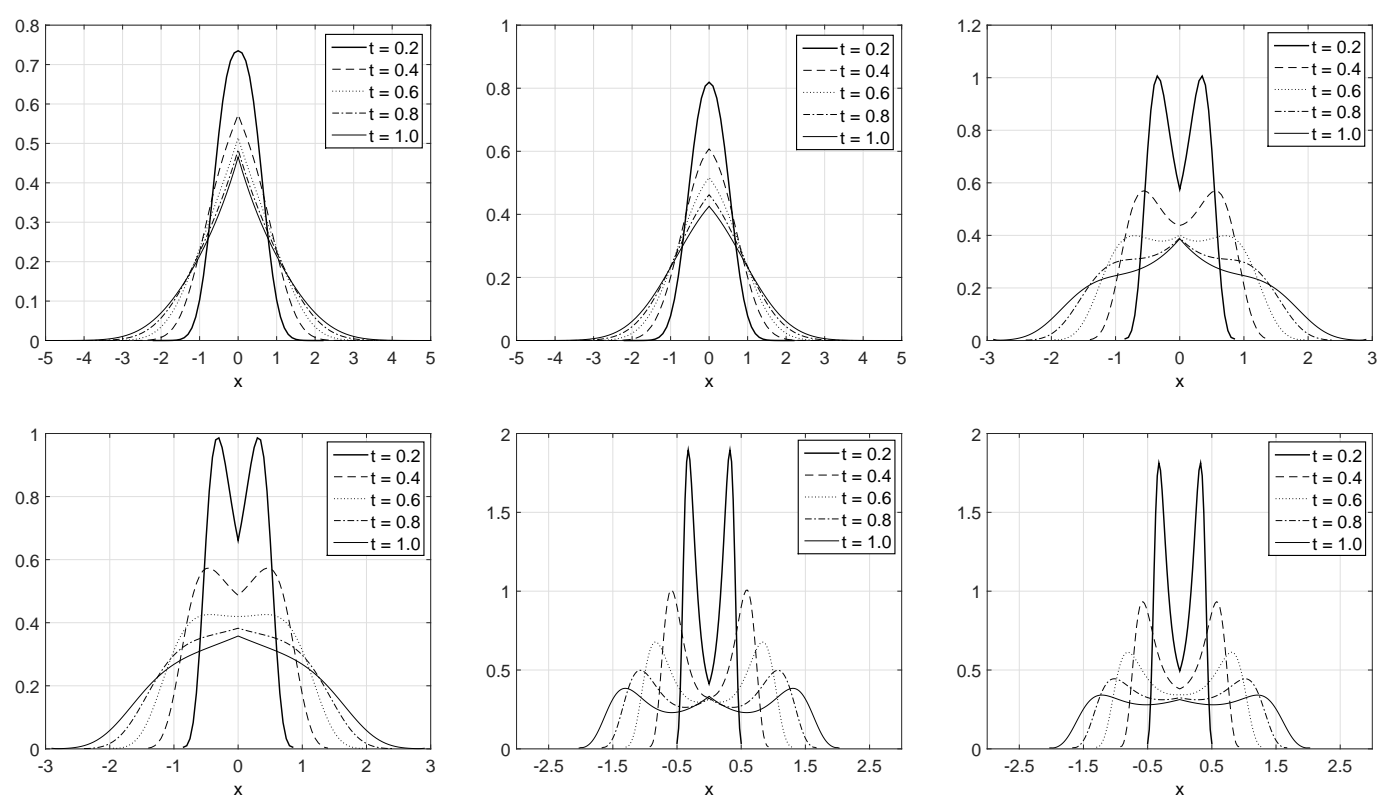

Figure 6: Plots of the $\mathfrak{f}^{\dagger}$-component of $\mathfrak{G}_{1}^{\alpha, \beta}$ for the following pairs of fractional parameters $(\alpha, \beta):(0.2,1.2)$ $(0.6,1.2),(0.2,1.5)$ (1st line, from left to right), $(0.6,1.5),(0.2,1.7),(0.6,1.7)$ (2nd line, from left to right).

From Figures 4, 5, and 6 it is readily seen that with the increasing of time the behavior of the FS changes in the origin and the decay becomes slower. The behaviour of the extreme points is analogous to the case when $t=1$.

\subsection{Case $n=3$}

Considering $n=3$ and $a=c=1$ in (29) we obtain the following expression for $\mathfrak{G}_{3}^{\alpha, \beta}$ :

$$
\begin{aligned}
\mathfrak{G}_{3}^{\alpha, \beta}(x, t)= & \left(D_{x}+\mathfrak{f}^{\dagger}\right) \delta(x)-\frac{x}{4 \pi|x|^{3}}\left(\frac{1}{\Gamma(1-\beta) t^{\beta}}+\frac{1}{\Gamma(1+\alpha) t^{\alpha}}\right) \\
& +\frac{x}{4 \pi t^{2 \beta}|x|} \sum_{k=0}^{+\infty} \frac{\left(-t^{\beta-\alpha}\right)^{k}}{k !} \sum_{p=0}^{+\infty} \frac{\left(-\frac{p+4}{2}\right)_{k}}{(p+2) \Gamma\left(1-\frac{\beta(p+4)}{2}+(\beta-\alpha) k\right) p !}\left(-\frac{|x|}{t^{\frac{\beta}{2}}}\right)^{p} \\
& +\mathfrak{f}\left[\frac{1}{4 \pi t^{\beta}|x|}\left(\frac{1-2 \beta+t^{\beta}}{\Gamma(2-2 \beta) t^{\beta}}+\frac{1-\beta-\alpha+t^{\beta}}{\Gamma(2-\beta-\alpha) t^{\alpha}}\right)\right. \\
& \left.-\frac{1}{4 \pi t^{\frac{5 \beta}{2}}} \sum_{k=0}^{+\infty} \frac{\left(-t^{\beta-\alpha}\right)^{k}}{t^{k} k !} \sum_{p=0}^{+\infty} \frac{\left(-\frac{p+3}{2}\right)_{k}}{(p+1) !} \frac{1-\frac{\beta(p+5)}{2}+(\beta-\alpha) k}{\Gamma\left(2-\frac{\beta(p+5)}{2}+(\beta-\alpha) k\right)}\left(-\frac{|x|^{2}}{t^{\frac{\beta}{2}}}\right)^{p}\right] \\
& +\mathfrak{f}^{\dagger}\left[\frac{1}{4 \pi|x|}\left(\frac{1}{\Gamma(1-\beta) t^{\beta}}+\frac{t^{-\alpha}}{\Gamma(1-\alpha)}\right)\right. \\
& \left.-\frac{1}{4 \pi t^{\frac{3 \beta}{2}}} \sum_{k=0}^{+\infty} \frac{\left(-t^{\beta-\alpha}\right)^{k}}{k !} \sum_{p=0}^{+\infty} \frac{\left(-\frac{p+3}{2}\right)_{k}}{(p+1) \Gamma\left(1-(\beta-\alpha) k-\frac{\beta(p+3)}{2}\right) p !}\left(-\frac{|x|}{t^{\frac{\beta}{2}}}\right)^{p}\right]
\end{aligned}
$$

We show in Figures 7, 8 and 9 the graphical representation of $\mathfrak{G}_{3}^{\alpha, \beta}$. 

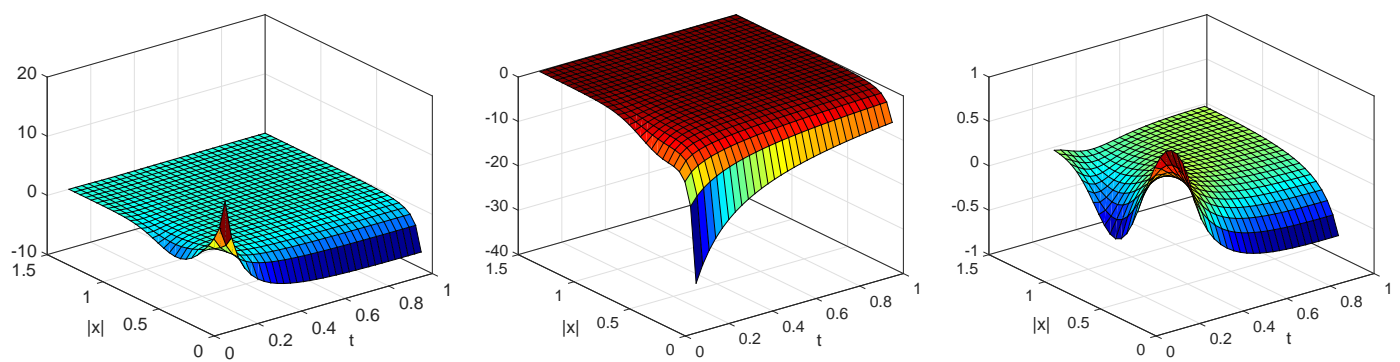

Figure 7: Plots of the vectorial-component of $\mathfrak{G}_{3}^{\alpha, \beta}$ for the following pairs of fractional parameters $(\alpha, \beta)$ : $(0.2,1.2)$ (left), $(0.8,1.2)$ (center), and $(0.4,1.4)$ (right).
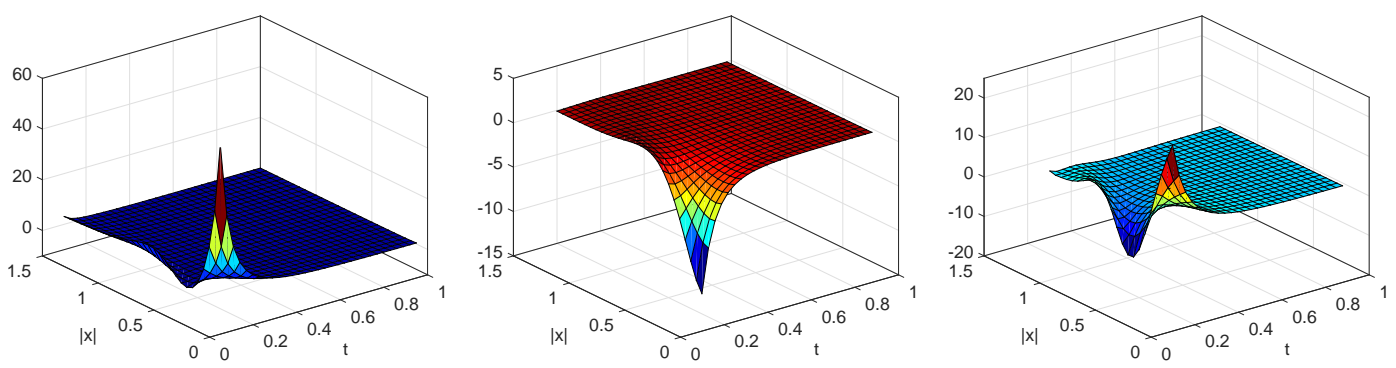

Figure 8: Plots of the $\mathfrak{f}$-component of $\mathfrak{G}_{3}^{\alpha, \beta}$ for the following pairs of fractional parameters $(\alpha, \beta):(0.2,1.2)$ (left), $(0.8,1.2)$ (center), and $(0.4,1.4)$ (right).
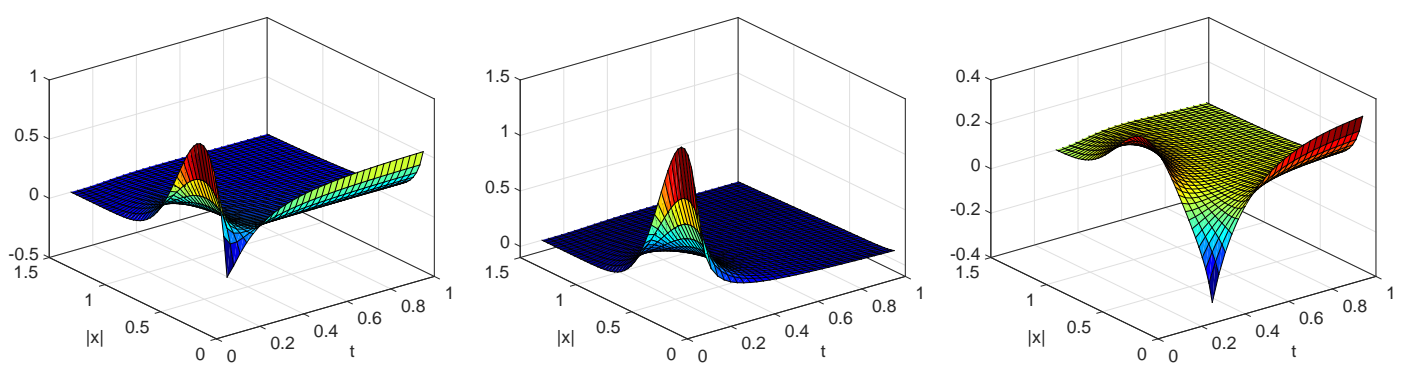

Figure 9: Plots of the $\mathfrak{f}^{\dagger}$-component of $\mathfrak{G}_{3}^{\alpha, \beta}$ for the following pairs of fractional parameters $(\alpha, \beta):(0.2,1.2)$ (left), $(0.8,1.2)$ (center), and $(0.4,1.4)$ (right).

For the three dimensional case we observe similar behaviours as in the case $n=1$. We also observe a different range of values of $G_{3}^{\alpha, \beta}$.

\section{Some Cauchy and Poisson problems}

The FS obtained previously can be used to solve some Cauchy and Poisson problems involving the time-fractional telegraph and telegraph Dirac operators studied in Sections 3 and 4.

Theorem 6.1 For $x \in \mathbb{R}^{n}, t>0, a \in \mathbb{R}_{0}^{+}, c \in \mathbb{R}^{+}, 0<\alpha \leq 1,1<\beta \leq 2, C_{0} \in \mathbb{R}$, and $h(x, t) \in L_{2}\left(\mathbb{R}^{n} \times \mathbb{R}^{+}\right)$ such that $\widehat{h}(\kappa, t)$ lies in $C_{-1}$ when $\beta=2$ or in $C_{-1}^{1}$ when $1<\beta<2$ for each $\kappa$, the solution of the Poisson problem

$$
\left\{\begin{array}{l}
\left(D_{t}^{\beta}+a D_{t}^{\alpha}-c^{2} \Delta_{x}\right) u(x, t)=h(x, t) \\
u(x, 0)=C_{0} \delta(x) \\
\frac{\partial u}{\partial t}(x, 0)=0
\end{array}\right.
$$


is given in the Fourier domain by

$$
\widehat{u}(\kappa, t)=\int_{0}^{t} s^{\beta-1} E_{(\beta-\alpha, \beta), \beta}\left(-a t^{\beta-\alpha},-c^{2}|\kappa|^{2} t^{\beta}\right) \hat{h}(\kappa, t-s) d s+C_{0} \widehat{G}_{n}^{\alpha, \beta}(\kappa, t) .
$$

Proof: Applying the Fourier transform in $\mathbb{R}^{n}$ to (33) we get the following initial-value problem:

$$
\left\{\begin{array}{l}
\left(D_{t}^{\beta}+a D_{t}^{\alpha}+c^{2}|k|^{2}\right) \widehat{u}(\kappa, t)=\hat{h}(\kappa, t) \\
\widehat{u}(\kappa, 0)=C_{0} \\
\frac{\partial \widehat{u}}{\partial t}(\kappa, 0)=0
\end{array} .\right.
$$

To solve the problem (34) we apply Theorem 2.8 with $\lambda_{1}=-a, \lambda_{2}=-c^{2}|\kappa|^{2}, \mu=\beta, \mu_{1}=\alpha, \mu_{2}=0, n=2$, $m=2, g(t)=\hat{h}(\kappa, t)$, and $k=1$, thus obtaining the following solution:

$$
\widehat{u}(\kappa, t)=\int_{0}^{t} s^{\beta-1} E_{(\beta-\alpha, \beta), \beta}\left(-a t^{\beta-\alpha},-c^{2}|\kappa|^{2} t^{\beta}\right) \hat{h}(\kappa, t-s) d s+C_{0} u_{0}(\kappa, t) .
$$

where

$$
u_{0}(\kappa, t)=\widehat{G}_{n}^{\alpha, \beta}(\kappa, t)=1-c^{2}|\kappa|^{2} t^{\beta} E_{(\beta-\alpha, \beta), 1+\beta}\left(-a t^{\beta-\alpha},-c^{2}|\kappa|^{2} t^{\beta}\right)
$$

Since the time-fractional telegraph and telegraph Dirac operators are linear with constant coefficients, and due to the properties of the convolution we obtain the following immediate results:

Theorem 6.2 For $x \in \mathbb{R}^{n}, t>0, a \in \mathbb{R}_{0}^{+}, c \in \mathbb{R}^{+}, 0<\alpha \leq 1,1<\beta \leq 2$, and $\varphi \in L_{2}\left(\mathbb{R}^{n}\right)$, the solution of the Cauchy problem

$$
\left\{\begin{array}{l}
\left(D_{t}^{\beta}+a D_{t}^{\alpha}-c^{2} \Delta_{x}\right) u(x, t)=0 \\
u(x, 0)=\varphi(x) \\
\frac{\partial u}{\partial t}(x, 0)=0
\end{array}\right.
$$

is given by

$$
u(x, t)=\int_{\mathbb{R}^{n}} G_{n}^{\alpha, \beta}(x-\xi, t) \varphi(\xi) d \xi
$$

Theorem 6.3 For $x \in \mathbb{R}^{n}, t>0, a \in \mathbb{R}_{0}^{+}, c \in \mathbb{R}^{+}, 0<\alpha \leq 1,1<\beta \leq 2$, and $\varphi \in L_{2}\left(\mathbb{R}^{n}\right)$, the solution of the Cauchy problem

$$
\left\{\begin{array}{l}
\left(c D_{x}+\mathfrak{f}\left(D_{t}^{\beta}+a D_{t}^{\alpha}\right)+\mathfrak{f}^{\dagger}\right) u(x, t)=0 \\
u(x, 0)=\varphi(x) \\
\frac{\partial u}{\partial t}(x, 0)=0
\end{array}\right.
$$

is given by

$$
u(x, t)=\int_{\mathbb{R}^{n}} \mathfrak{G}_{n}^{\alpha, \beta}(x-\xi, t) \varphi(\xi) d \xi
$$

Acknowledgement: The authors were supported by Portuguese funds through the CIDMA - Center for Research and Development in Mathematics and Applications, and the Portuguese Foundation for Science and Technology ("FCT-Fundaçâo para a Ciência e a Tecnologia"), within project UID/MAT/ 0416/2013. N. Vieira is Auxiliar Researcher, under the FCT Researcher Program 2014 (Ref: IF/00271/2014). 


\section{References}

[1] R.G. Buschman, H-functions of two variables III, Pure Appl. Math. Sci., 9, (1978), 13-18.

[2] R.G. Buschman, H-functions of two variables I, Indian J. Math., 20, (1978), 132-153.

[3] M. Caputo, Linear Models of Dissipation Whose Q Is Almost Frequency Independent-II, Geophys. J. R. Astron. Soc., 13-No.5, (1967), 529-539.

[4] P. Cerejeiras and N. Vieira, Regularization of the non-stationary Schrdinger operator, Math. Methods Appl. Sci., 32-No.5, (2009), 535-555.

[5] P. Cerejeiras and F. Sommen, Clifford algebra applied to the heat equation, in: More progresses in analysis. Proceedings of the 5th international ISAAC congress, H.G.W. Begehr et al (eds.), Catania, Italy, 25-30/07/2005, World Scientific, Hackensack, NJ, (2009), 1043-1050.

[6] P. Cerejeiras, U. Kähler and F. Sommen, Parabolic Dirac operators and the Navier-Stokes equations over timevarying domains, Math. Meth. in Appl. Sc., 28-No.14, (2005), 1715-1724.

[7] R. Delanghe, F. Sommen and V. Souček, Clifford algebras and spinor-valued functions. A function theory for the Dirac operator, Mathematics and its Applications-Vol.53, Kluwer Academic Publishers, Dordrecht etc., 1992.

[8] M. Ferreira and N. Vieira, Fundamental solutions of the time fractional diffusion-wave and parabolic Dirac operators, J. Math. Anal. Appl., 447-No.1, (2017), 329-353.

[9] M. Ferreira and N. Vieira, Eigenfunctions and fundamental solutions of the Caputo fractional Laplace and Dirac operators, Modern Trends in Hypercomplex Analysis, Trends in Mathematics Series, S. Bernstein, U. Khler, I. Sabadini, F.Sommen (Eds.), (2016), 191-202.

[10] M. Ferreira, and N. Vieira, Eigenfunctions and fundamental solutions of the fractional Laplace and Dirac operators: the Riemann-Liouville case, Complex Anal. Oper. Theory, 10-No.5, (2016), 1081-1100.

[11] M. Ferreira, M.M. Rodrigues and N. Vieira, Fundamental solution of the multi-dimensional time fractional telegraph equation, Fract. Calc. Appl. Anal., in press.

[12] C. Fox, The G and H functions as symmetrical Fourier kernels, Trans. Am. Math. Soc., 98, (1961), 395-429.

[13] K. Gürlebeck and W. Sprößig, Quaternionic and Clifford calculus for physicists and engineers, Mathematical Methods in Practice, Wiley, Chichester, 1997.

[14] H.J. Haubold, A.M. Mathai and R.K. Saxena, Mittag-Leffler functions and their applications, J. Appl. Math., (2011), Article ID 298628, 51 p.

[15] U. Kähler, and N. Vieira, Fractional Clifford analysis, Hypercomplex Analysis: New perspectives and applications, Trends in Mathematics, S. Bernstein, U. Kähler, I. Sabadini, and F. Sommen (eds.), Birkhäuser, Basel, (2014), 191-201.

[16] A. Kilbas, H.M. Srivastava and J.J. Trujillo, Theory and applications of fractional differential equations, NorthHolland Mathematics Studies-Vol.204, Elsevier, Amsterdam, 2006.

[17] A. Kilbas and M. Saigo, H-transforms. Theory and applications, Analytical Methods and Special Functions-Vol.9, Chapman \& Hall/CRC, Boca Raton, FL, 2004.

[18] Y. Luchko and R. Gorenflo, An operational method for solving fractional differential equations with the Caputo derivatives Acta Math. Vietnam., 24-No.2, (1999), 207-233.

[19] J. Lundgren, Convergence acceleration of alternating series, (https://www.mathworks.com/matlabcentral/ fileexchange/25200-altsum), MATLAB Central File Exchange, 2011. Access: 12/09/2016.

[20] E. Orsingher and L. Beghin, Time-fractional telegraph equations and telegraph processes with Brownian time, Probab. Theory Relat. Fields, 128-No.1, (2004), 141-160.

[21] I. Podlubny, Fractional Differential Equations. An introduction to fractional derivatives, fractional differential equations, to methods of their solution and some of their applications, Mathematics in Science and Engineering 198, Academic Press, San Diego, 1999.

[22] N. Vieira, Fischer Decomposition and Cauchy-Kovalevskaya extension in fractional Clifford analysis: the RiemannLiouville case, Proc. Edinb. Math. Soc., II. Ser., 60-No.1, (2017), 251-272. 